\title{
Helioseismic and neutrino data-driven reconstruction of solar properties
}

\author{
Ningqiang Song, ${ }^{1 \star}$ M. C. Gonzalez-Garcia, ${ }^{1,2,3}$ Francesco L. Villante,,${ }^{4,5}$ \\ Nuria Vinyoles ${ }^{6,7}$ and Aldo Serenelli ${ }^{6,7 \star}$ \\ ${ }^{1}$ C.N. Yang Institute for Theoretical Physics, State University of New York at Stony Brook, Stony Brook, NY 11794-3840, USA \\ ${ }^{2}$ Departament de Fisíca Quàntica i Astrofísica and Institut de Ciencies del Cosmos, Universitat de Barcelona, Diagonal 647, E-08028 Barcelona, Spain \\ ${ }^{3}$ Institució Catalana de Recerca i Estudis Avançats (ICREA), Pg. Lluis Companys 23, E-08010 Barcelona, Spain \\ ${ }^{4}$ Dipartimento di Scienze Fisiche e Chimiche, Università dell'Aquila, I-67100 L'Aquila, Italy \\ ${ }^{5}$ Laboratori Nazionali del Gran Sasso (LNGS), Istituto Nazionale di Fisica Nucleare (INFN), I-67100 Assergi (AQ), Italy \\ ${ }^{6}$ Institute of Space Sciences (ICE, CSIC), Campus UAB, Carrer de Can Magrans s/n, E-08193 Bellaterra, Spain \\ ${ }^{7}$ Institut d'Estudis Espacials de Catalunya, Edificio Nexus, Carrer Gran Capità 2-4, E-08034 Barcelona, Spain
}

Accepted 2018 February 28. Received 2018 February 24; in original form 2017 October 5

\begin{abstract}
In this work, we use Bayesian inference to quantitatively reconstruct the solar properties most relevant to the solar composition problem using as inputs the information provided by helioseismic and solar neutrino data. In particular, we use a Gaussian process to model the functional shape of the opacity uncertainty to gain flexibility and become as free as possible from prejudice in this regard. With these tools we first readdress the statistical significance of the solar composition problem. Furthermore, starting from a composition unbiased set of standard solar models (SSMs) we are able to statistically select those with solar chemical composition and other solar inputs which better describe the helioseismic and neutrino observations. In particular, we are able to reconstruct the solar opacity profile in a data-driven fashion, independently of any reference opacity tables, obtaining a 4 percent uncertainty at the base of the convective envelope and 0.8 percent at the solar core. When systematic uncertainties are included, results are 7.5 per cent and 2 per cent, respectively. In addition, we find that the values of most of the other inputs of the SSMs required to better describe the helioseismic and neutrino data are in good agreement with those adopted as the standard priors, with the exception of the astrophysical factor $S_{11}$ and the microscopic diffusion rates, for which data suggests a 1 per cent and 30 per cent reduction, respectively. As an output of the study we derive the corresponding data-driven predictions for the solar neutrino fluxes.
\end{abstract}

Key words: neutrinos - Sun: abundances - Sun: helioseismology - Sun: interior.

\section{INTRODUCTION}

Standard solar models (SSMs; Bahcall \& Ulrich 1988; TurckChieze et al. 1988; Bahcall \& Pinsonneault 1992, 1995; Bahcall, Pinsonneault \& Basu 2001; Bahcall, Serenelli \& Basu 2005b; PeñaGaray \& Serenelli 2008; Serenelli, Haxton \& Pena-Garay 2011; Vinyoles et al. 2017) describe the Sun present-day properties as a result of its evolution starting at the pre-main sequence. A set of observational parameters are taken as constraints that SSMs calculations have to satisfy by construction. They include the present surface abundances of heavy elements and surface luminosity of the Sun, as well as its age, radius, and mass. The modelling relies on some simplifying assumptions such as hydrostatic equilib-

^E-mail: ningqiang.song@stonybrook.edu (NS); aldos@ice.csic.es (AS) rium, spherical symmetry, homogeneous initial composition, and evolution at constant mass. SSMs have been constantly refined by including updated experimental results and observations regarding the values in physical input parameters. Examples include the values of the nuclear reaction rates and the surface abundances. There have also been improvements on the accuracy of the calculation of the constituent quantities like the equation of state and the radiative opacity, as well as the inclusion of new physical effects like the diffusion of elements.

The Sun burns, i.e. it generates power through nuclear fusion, with the basic energy source being the burning of four protons into an alpha particle, two positrons, and two neutrinos. Being only weakly interacting, the neutrinos can exit the Sun relatively unaffected. Thus, they give us the opportunity to learn about the solar interior and test in an almost direct way our understanding of nuclear energy production in the solar core (Bahcall 1964). With this 
objective the original neutrino experiments were designed, their goal being somewhat diverted by the appearance of the then called 'solar neutrino problem' (Bahcall, Bahcall \& Shaviv 1968; Bahcall \& Davis 1976). Thanks to the increasing experimental accuracy of the measured neutrino flux in radiochemical experiments Chlorine (Cleveland et al. 1998), Gallex/GNO (Kaether et al. 2010), and SAGE (Abdurashitov et al. 2009), together with the upcoming of the real-time experiments, Super-Kamiokande (Hosaka et al. 2006; Cravens et al. 2008; Abe et al. 2011; Koshio 2015), SNO (Aharmim et al. 2013), and Borexino (Bellini et al. 2010, 2011, 2014a,b), we have now reached the solution of the problem. It implied the modification of the Standard Model (of particle physics) with the addition of neutrino masses and leptonic mixing which imply both flavour transition of the solar neutrinos from production to detection (Pontecorvo 1968; Gribov \& Pontecorvo 1969), and non-trivial effects in their flavour evolution when crossing dense regions of matter, the so-called LMA-MSW flavour transitions (Wolfenstein 1978; Mikheev \& Smirnov 1985).

Moreover the Sun beats. The discovery of solar oscillations (Leighton, Noyes \& Simon 1962; Noyes \& Leighton 1963) of global nature (Deubner 1975), and their interpretation as non-radial standing acoustic waves, or p modes, (Ulrich 1970) opened up the possibility to pierce the surface layers of the Sun and to probe its interior structure. A range of helioseismic techniques makes use of the observed frequency pattern to offer powerful insights as well (for example, see Basu \& Antia 2008). In particular, the sound speed as a function of depth can be reconstructed to precision of order 0.1 per cent. The transition from radiative to convective energy transport, or abrupt changes in the solar thermal structure from ionization, induce acoustic glitches that can be precisely localized; thus, with that level of precision it is possible to infer the depth of the convective envelope with 0.2 per cent accuracy and the surface helium abundance with 1.5 per cent accuracy. This is, the solar structure is well constrained and the Sun can be used as a solid benchmark for stellar evolution and as a laboratory for fundamental physics (see e.g. Fiorentini, Ricci \& Villante 2001; Bottino et al. 2002; Ricci \& Villante 2002; Gondolo \& Raffelt 2009; Vinyoles et al. 2015; Vinyoles \& Vogel 2016).

As usual in the history of physics, better experimental information opens up new questions. So in parallel to the increased precision on both solar neutrino detection and helioseismic solar results, a new puzzle emerged in the consistency of SSMs (Bahcall et al. 2005a). SSMs built in the last decade of the last century had notable successes in predicting the helioseismology-related measurements (Bahcall \& Pinsonneault 1992, 1995; Christensen-Dalsgaard et al. 1996; Bahcall et al. 2001, 2005b). A key element to this agreement was the input value of the abundances of heavy elements on the surface of the Sun used to compute SSMs (Grevesse \& Sauval 1998, hereafter GS98). But in the second half of the first decade of the 21 st century new determinations of these abundances became available. The new spectroscopic analysis from Asplund, Grevesse \& Sauval (2006); Asplund et al. (2009, hereafter AGSS09) pointed towards lower values by up to 40 percent. The SSMs built incorporating such lower metallicities failed at explaining the helioseismic inferences on solar structure (Bahcall et al. 2005a). The more moderate reductions found by Caffau et al. (2011) also led to a degraded agreement between solar models and helioseismic observations, although not as dramatic as in the case of AGSS09 solar abundances.

So far there has not been a successful solution of this puzzle as no obvious changes in the Sun modelling have been found which could be able to account for this discrepancy (Castro, Vauclair \& Richard 2007; Guzik \& Mussack 2010; Serenelli, Haxton \&
Pena-Garay 2011). This has led to the construction of two different sets of SSMs, one based on the older solar abundances (Grevesse \& Sauval 1998) implying high metallicity, and one assuming lower metallicity as inferred from the 'newer' determinations of the solar abundances (Asplund et al. 2006, 2009). In a subsequent set of works (Serenelli et al. 2009, 2011; Vinyoles et al. 2017), the neutrino fluxes and helioseismic predictions corresponding to such two models were detailed, based on updated versions of the solar model calculations.

Alternatively, attempts to use the information from helioseismic and neutrino observations to better determine the solar chemical composition and other solar properties started to be put forward (Delahaye \& Pinsonneault 2006; Villante et al. 2014). The technical complication arises from the fact that both neutrino and helioseismic results are outputs of the SSM simulations while chemical composition and the other properties to be inferred are inputs. We are faced then with a common issue in multivariable analysis, the consistent estimation of the values of input parameters (some even with unknown functional dependence) which can provide a valid set of outputs within a given statistical level of agreement with some data. Before the advent of fast computing facilities this could only be attempted by partially reducing the number of inputs to be allowed to vary. For example, in Villante et al. (2014) the problem was analysed in terms of three continuous multiplicative factors (the abundance of volatiles, that of refractories and that of $\mathrm{Ne}$ ) to parametrize the allowed departures of the SSM inputs from the adopted priors of the two model versions. Furthermore, for the opacity profile - an input which is not a parameter but a function - assumptions about its functional form and allowed range of functional variation had to be assumed.

In this work, we take a step forward in this program by making use of Bayesian inference methods applied with specific numerical tools such as MULTinest (Feroz \& Hobson 2008; Feroz, Hobson \& Bridges 2009; Feroz et al. 2013) which have been developed precisely to make such inference in large parameter spaces which may contain multiple modes and pronounced degeneracies. Furthermore we introduce the use of Gaussian process (GP), a non-parametric regression method, to reconstruct the opacity profile and its uncertainty without assuming a specific functional form.

The outline of the paper is as follows. In Section 2, we present a brief introduction to the Bayesian parameter inference methods which we are using in this work. Section 3 discusses the issues arising in the parametrization of the opacity profile function for which we first describe the traditional linear form in Section 3.1. Section 3.2 presents the alternative non-parametric GP method to reconstruct the opacity profile (see also Appendix $\mathrm{C}$ where we introduce the main concepts in GP method for non-parametric functional reconstruction). In Section 4, we first apply this methodology to readdress the solar composition problem by evaluating a test of significance of the two B16 SSMs (Vinyoles et al. 2017) and using the two prescriptions of the profiles of the opacity and its uncertainty (linear and GP). We find, as expected, that allowing for the most flexible GP form of the opacity uncertainty profile decreases the evidence against the B16-AGSS09met model but it is still strongly disfavoured. Section 5 contains our evaluation of the optimum solar composition, opacity profile, and other solar parameters, to describe the helioseismic and neutrino data by Bayesian inference starting with a composition unbiased set of SSMs. As an output of the study we derive the corresponding prediction for the solar neutrino fluxes. Finally, in section 6, we summarize our conclusions. Details of the construction of the Likelihood function used in the analysis of the helioseismic and neutrino data are summarized in Appendix B. 
Table 1. Jeffrey's scale for interpretation of the Bayes factors.

\begin{tabular}{lc}
\hline$\left|\ln \left(\mathcal{B}_{i j}\right)\right|$ & Strength of evidence \\
\hline$<1.0$ & Inclusive \\
$1.0-2.5$ & Weak to moderate \\
$2.5-5$ & Moderate to strong \\
$>5.0$ & Strong to very strong/decisive
\end{tabular}

\section{STATISTICAL FRAMEWORK}

Bayesian inference methods provide a consistent approach to the estimation of a set of parameters $\Theta$ in a model $M$ for the data $\boldsymbol{D}$. Bayes' theorem states that under the assumption that a model $M$ is true, complete inference of its parameters is given by the posterior distribution,

$\operatorname{Pr}(\boldsymbol{\Theta} \mid \boldsymbol{D}, M)=\frac{\operatorname{Pr}(\boldsymbol{D} \mid \boldsymbol{\Theta}, M) \operatorname{Pr}(\boldsymbol{\Theta} \mid M)}{\operatorname{Pr}(\boldsymbol{D} \mid M)}=\frac{\mathcal{L}(\boldsymbol{\Theta}) \pi(\boldsymbol{\Theta})}{\mathcal{Z}}$,

where $\mathcal{L}(\boldsymbol{\Theta}) \equiv \operatorname{Pr}(\boldsymbol{D} \mid \Theta, M)$ is the likelihood function. The prior probability density of the parameters is given by $\pi(\Theta) \equiv \operatorname{Pr}(\Theta \mid M)$, and should always be normalized, i.e. it should integrate to unity. Conversely the evidence $\mathcal{Z}_{i}=\operatorname{Pr}\left(\boldsymbol{D} \mid M_{i}\right)$ is the likelihood for the model quantifying how well the model describes the data.

From the posterior distribution one can construct reparametrization invariant Bayesian credible intervals by defining the "credible level' of a value $\eta=\eta_{0}$ of a subset of parameters simply as the posterior volume within the likelihood of that value,

$\mathrm{CL}\left(\eta_{0}\right)=\int_{\mathcal{L}(\eta)>\mathcal{L}\left(\eta_{0}\right)} \operatorname{Pr}(\eta \mid \boldsymbol{D}) \mathrm{d} \eta$.

This function can be converted to the 'number of $\sigma \mathrm{s}$ ' in the usual manner as

$S=\sqrt{2} \operatorname{erfc}^{-1}(1-\mathrm{CL})$.

Bayesian statistics is mostly suited to make a relative statement about the plausibility of a given model $M_{i}$ versus another $M_{j}$ by comparing their respective posterior probabilities. This is quantified by means of the Bayes factor

$\mathcal{B}_{i j}=\frac{\mathcal{Z}_{i}}{\mathcal{Z}_{j}}$

which is the ratio of evidences. Jeffrey's scale is often used for the interpretation of the Bayes factors (see Table 1). This gives what the ratio of posterior probabilities for the models would be if the overall prior probabilities for the two models were equal. Or in other words it shows by how much the probability ratio of model $M_{i}$ to model $M_{j}$ changes in the light of the data, and thus can be viewed as a numerical measure of evidence supplied by the data in favour of one hypothesis over the other.

It is also possible to make an absolute test of significance of a given model $M$ by using the prior predictive distribution, which is to be understood as a distribution of the possible observable outputs $\mathcal{O}$,

$$
\operatorname{Pr}(\mathcal{O})=\int \operatorname{Pr}(\mathcal{O} \mid \Theta) \pi(\Theta) \mathrm{d}^{N} \Theta
$$

to determine the probability distribution function for some statistics $\mathcal{T}(\mathcal{O})$ and compare it with what was actually observed. This would be done as usual by calculating the $p$-value

$p=\operatorname{Pr}\left(\mathcal{T}(\mathcal{O}) \geq \mathcal{T}\left(\mathcal{O}^{\text {dat }}\right)\right)$.
Table 2. Values of the 11 model independent input parameters and their prior uncertainties. Astrophysical $S$-factors (nuclear rates), solar age, and luminosity are shown in units of $\mathrm{MeV}$ b, year, and erg $\mathrm{s}^{-1}$, respectively.

\begin{tabular}{lcc}
\hline Quantity & Central value & $\sigma$ ( per cent) \\
\hline$S_{11}$ & $4.03 \times 10^{-25}$ & 1 \\
$S_{17}$ & $2.13 \times 10^{-5}$ & 4.7 \\
$S_{114}$ & $1.59 \times 10^{-3}$ & 7.5 \\
$S_{33}$ & 5.21 & 5.2 \\
$S_{34}$ & $5.6 \times 10^{-4}$ & 5.2 \\
$S_{e 7}$ & Equation (40) in Adelberger et al. (2011) & 2.0 \\
$S_{116}$ & $1.06 \times 10^{-2}$ & 7.6 \\
$S_{\text {hep }}$ & $8.6 \times 10^{-20}$ & 30.2 \\
$t_{\odot}$ & $4.57 \times 10^{9}$ & 0.44 \\
Diffusion & 1.0 & 15.0 \\
$L_{\odot}$ & $3.8418 \times 10^{33}$ & 0.4 \\
\hline
\end{tabular}

In this work we use multinest (Feroz \& Hobson 2008; Feroz et al. 2009; Feroz et al. 2013), a Bayesian inference tool which, given the prior and the likelihood, calculates the evidence with an uncertainty estimate, and generates posterior samples from distributions that may contain multiple modes and pronounced (curving) degeneracies in high dimensions.

The general procedure which we will follow is to use MC generated sets of SSMs obtained for different choices of the model input parameters. These are 20 quantities: the solar luminosity $-L_{\odot}$, the microscopic diffusion rate, the solar age $-t_{\odot}$, eight nuclear rates $-S_{11}, S_{33}, S_{34}, S_{17}, S_{e 7}, S_{114}, S_{\text {hep }}, S_{116}$, and nine element abundances - C, N, O, Ne, Mg, Si, S, Ar, Fe. We have not assumed any correlation between the element abundances for two reasons. Spectroscopic studies do not provide an assessment of such correlations (although systematic effects, for example stemming from the solar model atmosphere, must be present). Secondly, by assuming no prior correlation we guarantee that the posterior distributions will be less correlated. Statistically this is the least restrictive condition and gives the most flexibility for accomodating the helioseismic and solar neutrino data. We note that, as customarily in solar models, we adopt the meteoritic scale for abundances of refractory elements. The matching between the meteoritic and the photospheric scale introduces very small errors, as it is discussed in Appendix A. Finally, one must also input some parametrization of the Opacity profile and its uncertainty (more below).

The sets of models are generated according to priors for these inputs which reflect our knowledge of those (knowledge which is independent of the data used in our analysis). Generically the priors are assumed to be Gaussian distributed. The numerical values for the mean and standard distributions for the first 20 inputs are given in Vinyoles et al. (2017) and for convenience we reproduce them in Tables 2 and 5. The assumed priors for the first 11 inputs (see Table 2) are common to all models generated, while for the abundances there are two different sets of priors corresponding to high- $Z$ and low- $Z$ compositions leading to the B16-GS98 and B16AGSS09met model subsets, respectively (see Table 5).

Following the procedure outlined above we confront these models with the data from helioseismology and neutrino oscillation experiments described in Appendix B. They amount to an effective number of 32 data points from helioseismic data plus a large number of points from the global analysis of neutrino oscillation data used in Bergström et al. (2016) with which we build the likelihood 
function

$$
-2 \ln \mathcal{L}(\Theta)=\sum_{i, j} \frac{\left(\mathcal{O}_{i}^{\text {mod }}(\boldsymbol{\Theta})-\mathcal{O}_{i}^{\text {dat }}\right)}{\sigma_{i}^{\text {dat }}}\left(\rho^{\mathrm{dat}}\right)_{i j}^{-1} \frac{\left(\mathcal{O}_{j}^{\text {mod }}(\boldsymbol{\Theta})-\mathcal{O}_{j}^{\text {dat }}\right)}{\sigma_{j}^{\text {dat }}},
$$

where $\mathcal{O}_{i}^{\text {mod }}(\Theta)$ are the model predicted values for all these observables obtained by MC generation for a given set of values of the model inputs $\Theta$. The correlation matrix $\rho_{i j}^{\text {dat }}=\delta_{i j}$ for $i, j=1,32$ but it is not diagonal for all the other entries which correspond to the neutrino oscillation data. Effectively the neutrino oscillation part of the likelihood can be approximated by eight data points corresponding to the extracted solar flux normalizations given in the last column in Table B1 and with the correlation matrix in equation (B1).

With these likelihood functions we can obtain the posterior distribution for some (or all) of the input parameters. These posterior distributions will quantify how the inclusion of this additional data affects our knowledge of those properties of the Sun.

Also, as described above, we can use the prior predictive distribution corresponding to the two variants of the SSMs to carry out a test of significance and to obtain their corresponding $p$-values. For this, we define the statistical test $\mathcal{T}(\mathcal{O})$ (where $\mathcal{O}$ is an $n$-dimensional vector containing possible values for the $n$ observables):

$\left.\left.\mathcal{T}(\mathcal{O})=\left(\mathcal{O}-\overrightarrow{\langle} \mathcal{O}^{\text {mod }}\right\rangle\right)^{T}\left(C_{\text {dat }}+C_{\text {mod }}\right)^{-1}\left(\mathcal{O}-\overrightarrow{\langle} \mathcal{O}^{\text {mod }}\right\rangle\right)$,

where $C_{\text {dat }, i j}=\rho_{i j}^{\text {dat }} \sigma_{i}^{\text {dat }} \sigma_{j}^{\text {dat }}$ is the covariance matrix associated with the experimental uncertainties, and

$$
\begin{aligned}
C_{\text {mod }, i j} & =\left\langle\left(\mathcal{O}_{i}^{\text {mod }}-\overline{\mathcal{O}}_{i}^{\text {mod }}\right)\left(\mathcal{O}_{j}^{\text {mod }}-\overline{\mathcal{O}}_{j}^{\text {mod }}\right)\right\rangle \\
& \equiv\left\langle\left(\mathcal{O}_{i}^{\text {mod }}-\overline{\mathcal{O}}_{i}^{\text {mod }}\right)\right\rangle \rho_{\text {mod }, i j}\left\langle\left(\mathcal{O}_{i}^{\text {mod }}-\overline{\mathcal{O}}_{i}^{\text {mod }}\right)\right\rangle
\end{aligned}
$$

is the model covariance matrix obtained from the MC generated model predictions by sampling over the model input priors about their means $\overline{\mathcal{O}}_{i}^{\text {mod }} \equiv\left\langle\mathcal{O}_{i}^{\text {mod }}\right\rangle$.

The probability distribution of $\mathcal{T}(\mathcal{O})$ can be determined from the MC model predictions by generating pseudo-experimental results $\mathcal{O}$ normally distributed according to $C_{\text {dat }}$ around each $\mathcal{O}^{\text {mod }}$ in the MC generated model samples, and computing for each pseudoexperimental result the corresponding value of $\mathcal{T}$. We find that, as expected, the probability distribution of $\mathcal{T}(\mathcal{O})$ follows very closely a $\chi_{n}^{2}$-distribution.

Unlike the first 20 inputs listed above, the opacity profile is not a numerical parameter but a function. We describe next two different procedures to parametrize the uncertainty in its prior.

\section{TREATMENT OF THE RADIATIVE OPACITY}

A fundamentally important physical ingredient in solar models that cannot be quantified by just one parameter is the radiative opacity, which is a complicated function of temperature $(T)$, density $(\rho)$, and chemical composition of the solar plasma expressed here in terms of the helium $(Y)$ and heavy elements mass fractions $\left(Z_{i}\right.$, where $i$ runs over all metals included in opacity calculations). In our calculations, we take as a reference the atomic opacities from OP (Badnell et al. 2005) complemented at low temperatures by molecular opacities from Ferguson et al. (2005). The magnitude and functional form of its uncertainty is currently not well constrained in available opacity calculations. As a result, representation of the uncertainty in radiative opacity by a single parameter (Serenelli, Peña-Garay \& Haxton 2013) or by taking the difference between two alternative sets of opacity calculations (Bahcall, Serenelli \& Basu 2006; Villante et al. 2014) are strong simplifications, at best. In this paper, instead, we choose to follow a general and flexible approach based on opacity kernels originally developed by Tripathy \& Christensen-Dalsgaard (1998) and later on by Villante (2010), which we describe next.

The reference opacity calculation $\bar{\kappa}\left(\rho, T, Y, Z_{i}\right)$ can be modified by a generic function of $\mathrm{T}, \rho, Y$, and $Z_{i}$. For simplicity, we assume that opacity variations are parametrized as a function of $T$ alone such that

$\kappa\left(\rho, T, Y, Z_{\mathrm{i}}\right)=\left[1+\delta \kappa_{\mathrm{I}}(T)\right] \bar{\kappa}\left(\rho, T, Y, Z_{\mathrm{i}}\right)$

where $\delta \kappa_{\mathrm{I}}(T)$ is an arbitrary function that we call intrinsic opacity change. The Sun responds linearly even to relatively large opacity variations $\delta \kappa_{\mathrm{I}}(T)$ (Tripathy \& Christensen-Dalsgaard 1998; Villante 2010). Thus, the fractional variation of a generic SSM prediction

$\delta Q \equiv Q / \bar{Q}-1$

where $Q(\bar{Q})$ corresponds to the modified (reference) value, can be described as

$\delta Q=\int \frac{\mathrm{d} T}{T} K_{Q}(T) \delta \kappa_{\mathrm{I}}(T)$

by introducing a suitable kernel $K_{Q}(T)$ that describes the response of $Q$ to changes in the opacity at a given temperature. We determine the kernels $K_{Q}(T)$ numerically by studying the response of solar models to localized opacity changes as it was done in Tripathy \& Christensen-Dalsgaard (1998). Our results agree very well in all cases except for variations in the chemical composition because our models include gravitational settling.

The evaluation of $\delta Q$ is subject to the choice we make for $\delta \kappa_{\mathrm{I}}(T)$. In Haxton \& Serenelli (2008) and Serenelli et al. (2013) the opacity error was modelled as a 2.5 per cent constant factor at $1 \sigma$ level, comparable to the maximum difference between the OP and OPAL (Iglesias \& Rogers 1996) opacities in the solar radiative region. Villante (2010) showed that this prescription underestimates the contribution of opacity uncertainty to the sound speed and convective radius error budgets because the opacity kernels for these quantities are not positive definite and integrate to zero for $\delta \kappa(T)=$ const. Later on, Villante et al. (2014) considered the temperature-dependent difference between OP and OPAL opacities as $1 \sigma$ opacity uncertainty. However, it is by no means clear that this difference is a sensible measure of the actual level of uncertainty in current opacity calculations.

Based on the previous reasons, here we follow a different approach inspired by the most recent experimental and theoretical results and some simple assumptions. The contribution of metals to the radiative opacity is larger at the bottom of the convective envelope ( $\sim 70$ per cent) than at the solar core ( $\sim 30$ per cent). Moreover, at the base of the convective envelope, relevant metals like iron are predominantly in an L-shell configuration, for which atomic models are more uncertain than for the $\mathrm{K}$-shell configuration that predominates at solar core conditions. Also, in a recent theoretical analysis of line broadening modelling in opacity calculations, Krief, Feigel \& Gazit (2016) have found that uncertainties linked to it are larger at the base of the convective envelope than in the core. These arguments suggest that opacity calculations are more accurate at the solar core than in the region around the base of the convective envelope. It is thus natural to consider error parametrizations that allow opacity to fluctuate by a larger amount in the external radiative region than in the centre of the Sun. 


\subsection{Linear parametrization of intrinsic opacity profile uncertainty}

Taking all this into account, we consider the following parametrization for the intrinsic opacity change (relative to some reference value):

$\delta \kappa_{\mathrm{I}}(T)=a+b \frac{\log _{10}\left(T_{\mathrm{C}} / T\right)}{\tau}$

where $\tau=\log _{10}\left(T_{\mathrm{C}} / T_{\mathrm{CZ}}\right)=0.9, T_{\mathrm{C}}$ and $T_{\mathrm{CZ}}$ are the temperatures at the solar centre and at the bottom of the convective zone, respectively. This equation is applied only up to the lower regions of the convective envelope, where convection is adiabatic and changes in the opacity do not modify the solar structure. Opacity changes in the uppermost part of the convective envelope and atmosphere are absorbed in the solar calibration by changes in the mixing length parameter and, in sound speed inversions, by the surface term. In the context of SSMs, they will not produce changes in the solar properties considered in the present work.

Technically, the opacity uncertainty is incorporated in our model generation by extending the parameter space with two more independent inputs, $a$ and $b$, each with a Gaussian prior with zero mean and variances $\sigma_{a}$ and $\sigma_{b}$, respectively. This corresponds to assuming that the opacity error at the solar centre is $\sigma_{\min }=\sigma_{a}$, while it is given by $\sigma_{\text {out }} \simeq \sqrt{\sigma_{a}^{2}+\sigma_{b}^{2}}$ at the base of the convective zone. We fix $\sigma_{\text {in }}=\sigma_{a}=2$ per cent which is the average difference of the OP and OPAL opacity tables. This is also comparable to differences found with respect to the new OPAS opacity tables (Mondet et al. 2015) for the AGSS09 solar composition, the only one available in OPAS. The more recent OPLIB tables from Los Alamos (Colgan et al. 2016) show much larger differences in the solar core, about 1012 per cent lower than OP and up to 15 per cent lower than OPAS. However, OPLIB opacities lead to solar models that predict too low $Y_{\mathrm{S}}$ and $\Phi\left({ }^{7} \mathrm{Be}\right)$ and $\Phi\left({ }^{8} \mathrm{~B}\right)$ fluxes that cannot be reconciled with data. For $\sigma_{\text {out }}$ we choose 7 per cent (i.e. $\sigma_{b}=6.7$ per cent), motivated by the recent experimental results of Bailey et al. (2015) that have measured the iron opacity at conditions similar to those at the base of the solar convective envelope and have found a $7 \pm 4$ percent increase with respect to the theoretical expectations. The resulting prior for the intrinsic opacity profile uncertainty is shown in the upper left panel in Fig. 1 for both B16 models. Given the generated values for those two parameters we construct the function $\delta \kappa_{\mathrm{I}}(T)$ as in equation (13) and with that we compute the corresponding change in the output quantities as in equation (12).

As we will see below and was also discussed by Vinyoles et al. (2017), it turns out that our ad hoc linear parametrization of the intrinsic opacity uncertainty is not flexible enough to accommodate the tension between B16-AGSS09met model and data (especially sound speed data). This parametrization was chosen for its simplicity, whereas in fact the shape of the opacity uncertainty function is unknown. Thus, in the next section we turn to a more general modelling of the intrinsic opacity uncertainty based on a GP approach. A brief introduction of the general method is given in Appendix C.

\subsection{Gaussian process reconstruction of the opacity profile}

Our goal is to define the uncertainty of the opacity profile without using parametrized functions and to reconstruct the intrinsic opacity change $\delta \kappa_{\mathrm{I}}(T)$ that can lead to a better agreement with the data. In order to do this, following the discussion in Section 3.1, we assume that $\delta \kappa_{\mathrm{I}}(T)$ is a Gaussian variable with mean is $\mu_{P}(T) \equiv 0$ with a temperature-dependent variance $\sigma(T)$ which allows for 2 percent uncertainty in the solar centre and 7 percent at the base of the convective zone, i.e.

$\sigma(T)=0.02+(0.07-0.02) \frac{\log _{10}\left(T_{\mathrm{C}} / T\right)}{\tau}$.

As the values of the opacity at two different temperatures $T$ and $T^{\prime}$ may be not independent, we introduce a prior covariance function $C_{P}\left(T, T^{\prime}\right)$. A possible choice is

$C_{P}\left(T, T^{\prime}\right)=\sigma(T) \sigma\left(T^{\prime}\right) \rho\left(T, T^{\prime}\right)$,

with

$\rho\left(T, T^{\prime}\right)=\exp \left[-\frac{1}{2}\left(\frac{\log _{10} T-\log _{10} T^{\prime}}{\tau L}\right)^{2}\right]$.

Here, $L$ determines the characteristic correlation length over which $\delta \kappa_{\mathrm{I}}(T)$ can vary significantly and it is the only hyperparameter in our analysis. According to the above definition, $L=1$ means maximum correlation between the opacity at the edge of the convective zone and at the centre. If $L$ is too large the correlation is too strong and the model is over constrained. If, on the other hand, $L$ is too small, we are allowing opacity errors to dominate the output of solar models, and we can barely learn anything from the data. Moreover, there is a physically motivated lower bound for $L$ given by the temperature range over which the opacity can vary substantially. In the solar interior $|\partial \ln \kappa / \partial \ln T|<2$ (Colgan et al. 2016). From this, the smallest temperature range over which $\Delta \ln \kappa \approx 1$ is $\Delta \ln$ $T \approx 0.5$, i.e. $L_{\min } \approx 0.2$.

To implement the GP intrinsic opacity uncertainty in our analysis we start by choosing a set of $N$ points in which we evaluate the function $\delta \kappa_{\mathrm{I} i}=\delta \kappa_{\mathrm{I}}\left(T_{i}\right)$. We take $N=11$ and choose the points to be uniformly distributed in $\log _{10} T$ between 6.3 and 7.2. The parameter space for model generation is thus extended with 12 more input parameters: the length $L$ and the $11 \delta \kappa_{\mathrm{I} i}$ values. For the correlation length we assume a uniform prior in $\log _{10} L$ between $\log _{10} 0.2$ and $\log _{10} 1$. The values $\delta \kappa_{I i}$ are generated according to prior distribution defined by equations (14)-(16), together with $\mu_{P}(T) \equiv 0 .{ }^{1}$ Given a set of values for the $11 \delta \kappa_{\mathrm{I} i} \mathrm{~s}$ we construct the full function $\delta \kappa_{\mathrm{I}}(T)$ by linear interpolation between these values and with that we compute the corresponding change in the output predictions as in equation (12). The resulting prior distribution of the intrinsic opacity change is shown in the upper right panel of Fig. 1. As seen in the figure, the ranges of uncertainty profiles for the linear parametrization and the GP opacity are very similar. They do, however, lead to different conclusions when testing the SSM's models versus the data (in particular versus helioseismic data) as described in Section 4.

\subsection{The degeneracy between opacity and composition effects}

The properties of the Sun depend on its opacity profile $\kappa_{\mathrm{SSM}}(T)$ that we define as

$\kappa_{\mathrm{SSM}}(T)=\kappa\left(\rho(T), T, Y(T), Z_{i}(T)\right)$,

where $\rho(T), Y(T)$, and $Z_{i}(T)$ describe the density, helium, and heavy element abundance stratifications as a function of the temperature of the solar plasma in a given SSM. This is indeed the quantity that determines the efficiency of radiative energy transport and, thus, the

\footnotetext{
${ }^{1}$ We studied the number of points which maximized the smoothness of the output profile versus computing time and found that increasing $N$ beyond 11 did not yield any better results.
} 

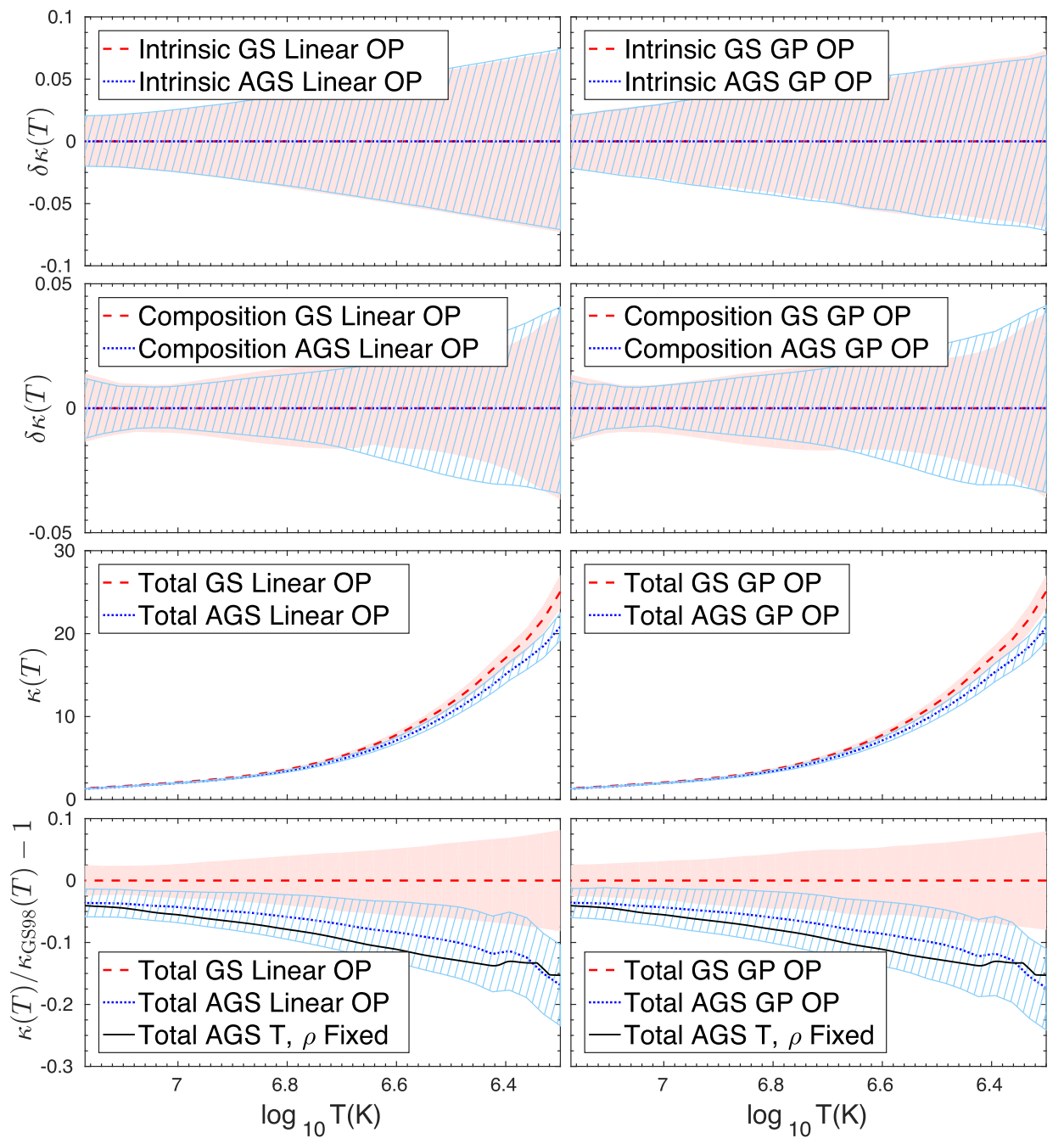

Figure 1. Priors for the intrinsic opacity change (upper panels), the composition opacity change, equation (20) (second row panels), and the total opacity profile in absolute value (third row panels) and relative to the central value for the GS98 model (lower panels). Left correspond to the linear parametrization of the intrinsic opacity uncertainty while right corresponds to the Gaussian process one. The lower panels also shows the curve (same in both panels) with the opacity difference due to the different compositions at fixed physical properties (the fixed values of $T$ and $\rho$ are those of the GS98 model so the reference GS98 opacity profile is common to all curves in the lower panels)

temperature gradient at each point of the Sun and that is plotted in the panels in the third row of Fig. 1 for both B16 models. To better compare the range of prior uncertainty of the opacity profile with the difference between the central values of the priors of the two SSMs, we plot in the lower panels of Fig. 1 the same total opacity variation relative to the central value of the B16-GS98 model. The fractional opacity difference due to the change of composition only, i.e. compared at fixed $T$ and $\rho$, is also shown for reference.

When considering an intrinsic opacity change $\delta \kappa_{\mathrm{I}}(T)$ and/or other input parameters in the SSMs are varied, the SSM needs to be recalibrated, thus obtaining different density and chemical abundance stratifications with respect to the reference SSM. As a consequence, the total variation of the solar opacity profile is given by

$$
\begin{aligned}
\delta \kappa_{\mathrm{SSM}}(T) \equiv & \kappa_{\mathrm{SSM}}(T) / \bar{\kappa}_{\mathrm{SSM}}(T)-1 \\
\simeq & \delta \kappa_{\mathrm{I}}(T)+\frac{\partial \ln \kappa}{\partial \ln \rho} \delta \rho(T)+\frac{\partial \ln \kappa}{\partial \ln Y} \delta Y(T) \\
& +\sum_{i} \frac{\partial \ln \kappa}{\partial \ln Z_{i}} \delta Z_{i}(T),
\end{aligned}
$$

where $\delta \rho(T), \delta Y(T)$, and $\delta Z_{i}(T)$ are the fractional variations of density and elemental abundances in the perturbed Sun with respect to the reference SSM, evaluated at a fixed temperature $T$.

As discussed above, the metal abundances $Z_{i}(T)$ are derived quantities that have to be obtained as a results of numerical solar modelling. However, when we consider a modification of the surface composition $\left\{z_{i}\right\}$, expressed here in terms of the quantities $z_{i} \equiv Z_{i, \mathrm{~S}} / X_{\mathrm{S}}$ where $Z_{i, \mathrm{~S}}$ is the surface abundance of the $i$-element and $X_{\mathrm{S}}$ is that of hydrogen, we can approximately assume $\delta Z_{i}(T) \simeq$ $\delta z_{i}$ where $\delta z_{i}$ is the fractional variation of $z_{i}$ with respect to some reference value. As a consequence, equation (18) can be rewritten as

$\delta \kappa_{\mathrm{SSM}}(T)=\delta \kappa_{\mathrm{I}}(T)+\delta \kappa_{\mathrm{Z}}(T)+\frac{\partial \ln \kappa}{\partial \ln \rho} \delta \rho(T)+\frac{\partial \ln \kappa}{\partial \ln Y} \delta Y(T)$

where the composition opacity change $\delta \kappa_{\mathrm{Z}}(T)$ is defined as

$\delta \kappa_{\mathrm{Z}}(T) \equiv \sum_{i} \frac{\partial \ln \kappa}{\partial \ln Z_{i}} \delta z_{i}$. 
Table 3. Comparison of B16 SSMs against different ensembles of solar observables.

\begin{tabular}{lccccccccc}
\hline & \multicolumn{2}{c}{ GS98 } & \multicolumn{2}{c}{ LIN-OP } & & \multicolumn{2}{c}{ GP-OP } \\
& & \multicolumn{2}{c}{ AGSS09met } & & GS98 & AGSS09met \\
\hline $\mathcal{O}$ & $n$ & $\mathcal{T}(\mathcal{O})$ & $p$-value $(\sigma)$ & $\mathcal{T}(\mathcal{O})$ & $p$-value $(\sigma)$ & $\mathcal{T}(\mathcal{O})$ & $p$-value $(\sigma)$ & $\mathcal{T}(\mathcal{O})$ & $p$-value $(\sigma)$ \\
$Y_{\mathrm{S}}+R_{\mathrm{CZ}}$ & 2 & 0.9 & 0.5 & 6.5 & 2.1 & 0.7 & 0.35 & 6.9 & 2.2 \\
$\delta c$ & 30 & 58.0 & 3.2 & 76.1 & 4.5 & 35.6 & 1.2 & 40.2 & 1.6 \\
All $v$-fluxes & 8 & 6.0 & 0.5 & 7.0 & 0.6 & 5.9 & 0.44 & 7.0 & 0.6 \\
Global & 40 & 65.0 & 2.7 & 94.2 & 4.7 & 45.1 & 1.1 & 57.1 & 2.1 \\
\hline
\end{tabular}

We define the total opacity change $\delta \kappa(r)$ as

$\delta \kappa(T)=\delta \kappa_{\mathrm{I}}(T)+\delta \kappa_{\mathrm{Z}}(T)$,

which groups together the contributions to $\delta \kappa_{\mathrm{SSM}}(T)$ directly related to the variations of the input parameters.

Note that metals have a negligible role in determining the equation of state of the solar plasma and in solar energy generation (except for carbon, nitrogen, and oxygen that determine the efficiency of the CNO cycle which is, however, largely subdominant in the Sun). Thus, the only structural effect produced by a modification of the surface composition $\left\{z_{i}\right\}$ is through the changes in the efficiency of radiative energy transport induced by the composition opacity change $\delta \kappa_{\mathrm{Z}}(T)$ defined above. In this respect, equation (21) although being approximate, is quite useful because it makes explicit the connection (and the degeneracy) between the effects produced by an intrinsic modification of the radiative opacity and those produced by a modification of the heavy element admixture. The physical quantity that drives the modification of the solar properties and that can be constrained by observational data is the total opacity change $\delta \kappa(T)$, not the intrinsic $\delta \kappa_{\mathrm{I}}(T)$ or the composition opacity change $\delta \kappa_{\mathrm{Z}}(T)$ separately.

For completeness, we show in the middle panels of Fig. 1 the prior distributions of the composition opacity changes for both B16-SSMs, calculated by considering the relative variations of the individual abundances $\delta z_{j}$ around their mean values for GS98 and AGSS09met surface compositions. The logarithmic derivatives $\partial \ln \kappa / \partial \ln Z_{i}$ can be found in the left-hand panel of fig. 10 in Villante et al. (2014). The prior distributions for $\delta \kappa_{\mathrm{Z}}(T)$ are identical in the left-hand and right-hand (middle) panels because the adopted procedure for describing the intrinsic opacity uncertainty does not alter the sampling in surface composition.

\section{TEST OF SIGNIFICANCE AND MODEL COMPARISON}

We start by performing a test of significance of the two B16 SSMs using the linear and GP models of the opacity uncertainty described in the previous section. Results are given in Table 3 where we show the value of the test statistics $\mathcal{T}$ in equation (8) for different combination of observables.

As seen from the table, global $p$-values are dominated by the sound speed for both models, although $Y_{\mathrm{S}}$ and $R_{\mathrm{CZ}}$ are also relevant for B16-AGSS09met. We also read from the table that when using the linear opacity uncertainty parametrization the global analysis yields a not too good $p$-value of $2.7 \sigma$ for B16-GS98 and considerably worse $(4.7 \sigma)$ for the B16-AGSS09met. The results are different when the GP opacity uncertainty is used which yields $p$-value of $1.1 \sigma$ and $2.1 \sigma$ for B16-GS98 and B16-AGSS09met, respectively.

In Fig. 2, we plot the fractional sound speed difference $\delta c(r) \equiv$ $\left(c_{\mathrm{obs}}(r)-c(r)\right) / c(r)$, where $c_{\mathrm{obs}}(r)$ is the sound speed inferred from helioseismic data, while $c(r)$ represents the sound speed profile predicted by the B16-GS98 (left) and B16-AGSS09met (right) model, respectively. The blue (lighter) hatched area and the red (darker) shaded area correspond to the $1 \sigma$ theoretical uncertainties in sound speed predictions obtained for linear and GP opacity uncertainty priors. As seen from the figure, and expected from the comparison of the top panels in Fig. 1, they are not very different in the two considered cases. Moreover, we observe from the figure that at almost all radii, independently of the adopted prescription, the sound speed profile of B16-GS98 fits well within the $1 \sigma$ data uncertainties. It may be thus surprising that the B16-GS98 model is not providing a good $p$-value in the case of linear opacity uncertainty parametrization.

The reason for the bad $p$-value obtained for the B16-GS98 model is that, as discussed in Vinyoles et al. (2017), changes in input quantities do not lead to variations in SSM sound speeds on very small radial scales, so values of the sound speed at different radii in solar models are strongly correlated, i.e. the model correlation matrix $\rho_{\text {mod, } i j}$ in equation (9) is highly non-diagonal. This is shown in the lower panels of Fig. 2 where we graphically display the values for the entries of the correlation matrix between the predicted sound speeds at the 30 locations (the correlation matrix is the same for both B16 models). As seen in the figure, the characteristic correlation length (i.e. the distance $\left|r_{i}-r_{j}\right|$ over which correlations between the predicted values of the sound speeds are strong, say $\left|\rho_{\text {mod, } i j}\right|$ $\gtrsim 0.5$ ) is much larger for the linear opacity profile parametrization than for the GP profile.

The more flexible implementation of the opacity profile uncertainty provided by the GP procedure permits to obtain a better description of the observational data for both B16-GS98 and B16AGSS09met models. To illustrate this point, Fig. 3 shows the posterior distribution of $L$, the correlation length hyperparameter [equation (15)]. As seen from the figure, the best possible description of the data is achieved with correlation lengths of average $\langle L\rangle \sim 0.2$, i.e. close to the lowest value permitted by the adopted prior that allows for short-scale modifications of the sound speed profiles.

We finish this section by giving in Table 4 the Bayes factors for the two models as obtained with their posterior probability distributions after including the neutrino and helioseismic data for the two assumed opacity profile uncertainties. From the table we conclude that the B16-AGSS09met models are always somewhat disfavoured with respect to the B16-GS98 model by all data sets but the most statistical significant effect is driven by the sound speed profile data. This is particularly the case for the linear opacity uncertainty profile for which the Bayes factor of -14.7 is enough for rejection of the model. Allowing for the most flexible GP form of the opacity uncertainty decreases the evidence against the B16-AGSS09met model to close to strong. In this respect, we notice that our assumption that the experimental errors of the 30 sound speed data points are uncorrelated is the most conservative hypothesis for the statistical 


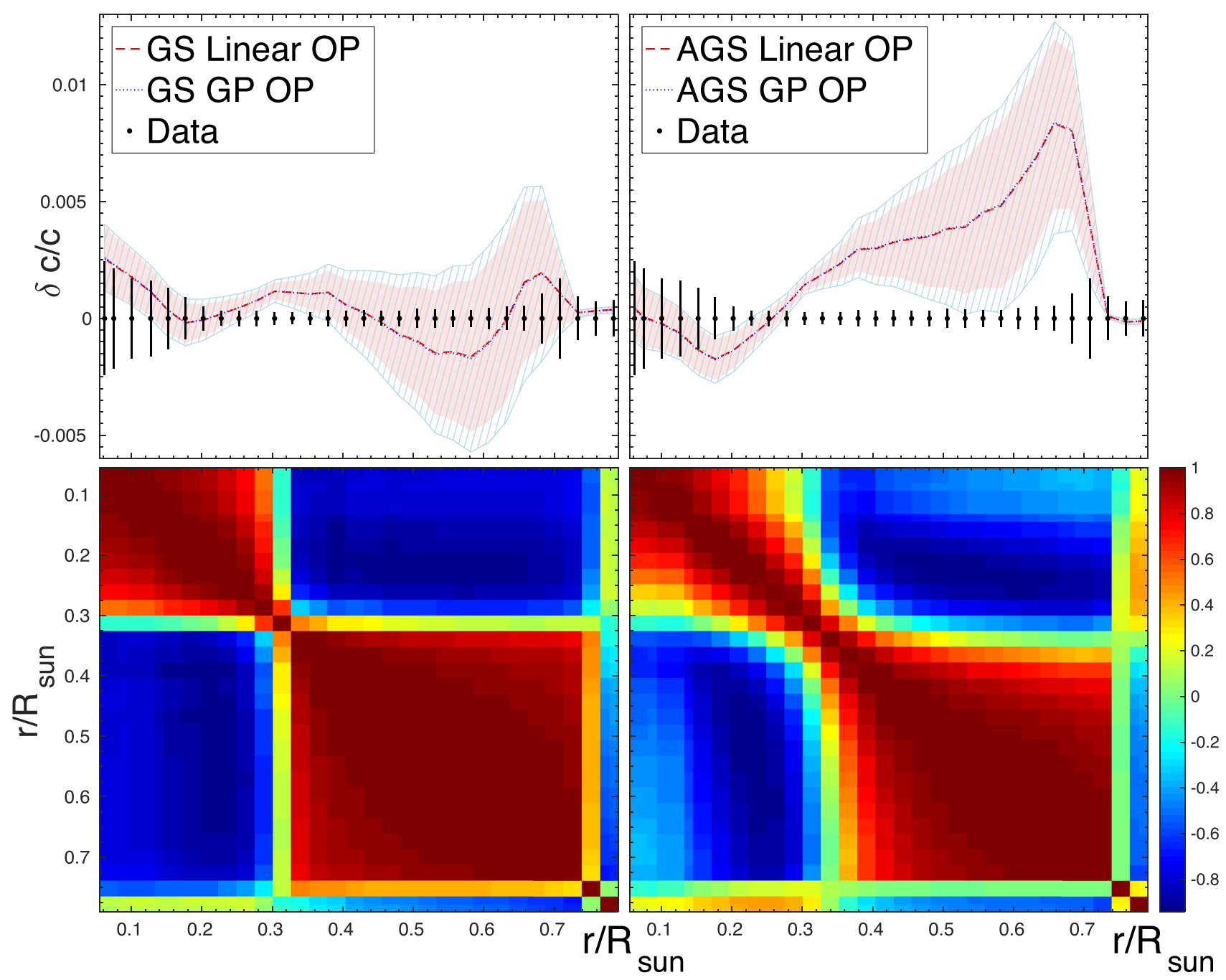

Figure 2. $1 \sigma$ range of variation of the fractional sound speed profiles as predicted by the priors the B16 SSM models and for both opacity profile priors discussed in the text (upper panels) compared with the 30 data points used in the analysis. The lower panels graphically display the values of the entries in the $30 \times 30$ model correlation matrix between the predicted sound speeds at the 30 points (which are the same for B16-GS98 and B16-AGSS09met models) for the linear opacity uncertainty parametrization (left) and the GP opacity uncertainty(right).

tests presented. It leads to the most favourable conclusion of the significant test for each model (ie to its lowest $p$-value) and to the weakest discrimination power between the two models (ie to the smallest module of the Bayes factor).

\section{DETERMINATION OF THE OPTIMUM COMPOSITION AND OPACITY PROFILE}

We now turn to the determination of the optimum solar composition which best describes the helioseismic and neutrino data. In order to do so we perform Bayesian parameter inference by using a top hat prior for the logarithmic abundances $\varepsilon_{i} \equiv \log _{10}\left(N_{i} / N_{\mathrm{H}}\right)+12$ that accommodates both the AGSS09met and GS98 admixtures, i.e. with value 1 between the $3 \sigma$ lower value of the AGSS09met composition and the $3 \sigma$ upper value of the GS998 composition for all the nine elements relevant for solar model construction given in Table 5, and zero outside this range. As before we study the dependence of our results on the two models for the opacity uncertainty.
We show in Fig. 4 the posterior probability distributions for the nine abundance parameters centred for reference around the GS98 ones, i.e. $\Delta \varepsilon_{j}=\varepsilon_{j}-\varepsilon_{j, \mathrm{GS} 98}$, and for the two choices of priors of the opacity uncertainties (linear or GP). The window for each abundance corresponds to the allowed range, i.e. where prior $=$ 1. Outside each window the value of the prior is zero. For the sake of comparison we also show in the figure the corresponding prior distributions for the B16-GS98 and B16-AGSS09met models. Notice that the distributions are given in arbitrary units and have been normalized in such a way that the maximum of all distributions lays at the same height to help comparison.

We list in the last two columns of Table 5 the corresponding $\pm 1 \sigma$ ranges for the logarithmic abundances $\varepsilon_{j}$ extracted from these posterior distribution. These can be compared with the determination of the same quantities in GS98 and AGSS09met compilations reported in the first two columns of the same table. From the figure and table we see that the available data are not capable of setting tight constraints on all the elements simultaneously. However, we find that the posterior for the combinations of $\mathrm{CNO}(\mathrm{C}+\mathrm{N}+\mathrm{O})$ and 


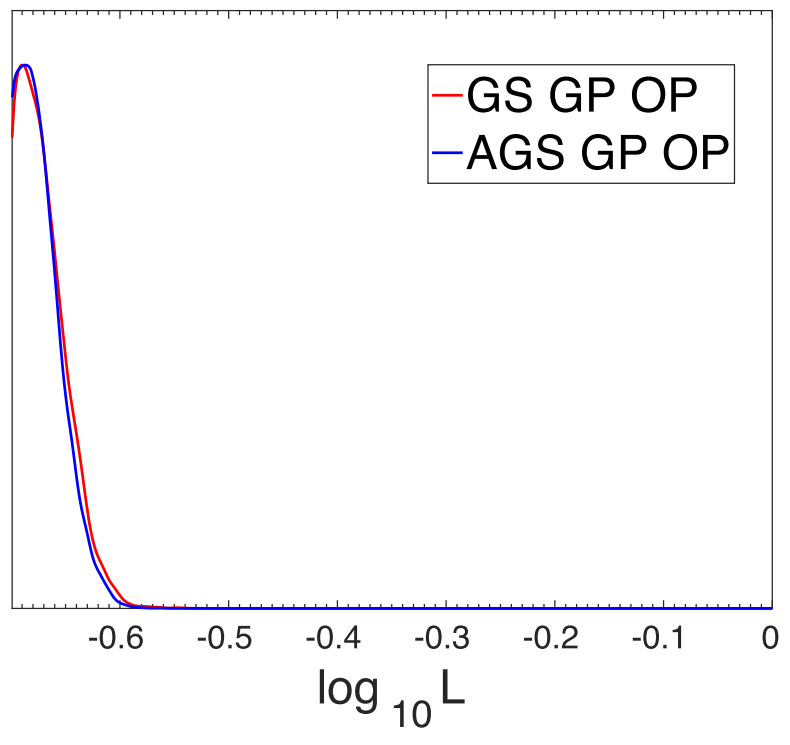

Figure 3. Posterior probability distribution for the correlation length hyperparameter $L$ of the GP opacity uncertainty for runs with the models B16-GS98 and B16-AGSS09met priors for the abundances.

Table 4. Bayes factor, $\ln (\mathcal{B})$, for the B16AGSS09met versus B16-GS98 model obtained with the different data sets (see Table 1 for interpretation).

\begin{tabular}{lcc}
\hline Data & \multicolumn{2}{c}{ B16-AGSS09met/B16-GS98 } \\
\hline & LIN-OP & GP-OP \\
$v$ & -0.23 & -0.27 \\
$+Y_{\mathrm{S}}+R_{\mathrm{CZ}}$ & -1.6 & -2.2 \\
+ Sound speeds & -14.7 & -4.1 \\
\hline
\end{tabular}

Table 5. $1 \sigma$ ranges for the logarithmic abundances $\varepsilon_{j}$. The first two columns show the mean values and uncertainties of the GS98 and AGSS09met heavy element admixtures. The last two columns give the ranges of the posterior distributions from the analysis of neutrino and helioseismic data for the two choices of the prior opacity uncertainties with uniform priors for the abundances.

\begin{tabular}{lcccc}
\hline Element & GS98 & AGSS09met & Linear & GP \\
\hline $\mathrm{C}$ & $8.52 \pm 0.06$ & $8.43 \pm 0.05$ & {$[8.32,8.56]$} & {$[8.31,8.51]$} \\
$\mathrm{N}$ & $7.92 \pm 0.06$ & $7.83 \pm 0.05$ & {$[7.88,8.10]$} & {$[7.81,8.05]$} \\
$\mathrm{O}$ & $8.83 \pm 0.06$ & $8.69 \pm 0.05$ & {$[8.82,8.91]$} & {$[8.80,8.94]$} \\
$\mathrm{Ne}$ & $8.08 \pm 0.06$ & $7.93 \pm 0.10$ & {$[7.87,8.06]$} & {$[7.90,8.16]$} \\
$\mathrm{Mg}$ & $7.58 \pm 0.01$ & $7.53 \pm 0.01$ & {$[7.54,7.60]$} & {$[7.52,7.58]$} \\
$\mathrm{Si}$ & $7.56 \pm 0.01$ & $7.51 \pm 0.01$ & {$[7.57,7.59]$} & {$[7.54,7.59]$} \\
$\mathrm{S}$ & $7.20 \pm 0.06$ & $7.15 \pm 0.02$ & {$[7.35,7.38]$} & {$[7.27,7.37]$} \\
$\mathrm{Ar}$ & $6.40 \pm 0.06$ & $6.40 \pm 0.13$ & {$[6.14,6.44]$} & {$[6.20,6.50]$} \\
$\mathrm{Fe}$ & $7.50 \pm 0.01$ & $7.45 \pm 0.01$ & {$[7.42,7.44]$} & {$[7.42,7.48]$} \\
$\mathrm{CNO}$ & $9.04 \pm 0.04$ & $8.92 \pm 0.03$ & {$[9.03,9.08]$} & {$[9.00,9.09]$} \\
Meteor. & $8.09 \pm 0.01$ & $8.04 \pm 0.01$ & {$[8.08,8.10]$} & {$[8.07,8.10]$} \\
\hline
\end{tabular}

meteorite $(\mathrm{Mg}+\mathrm{Si}+\mathrm{S}+\mathrm{Fe})$ abundances (Delahaye \& Pinsonneault 2006; Villante et al. 2014) have a comparable precision to GS98 and AGSS09met observational determinations for either choice of the opacity uncertainty parametrization. It is important to stress that, as mentioned above, these posterior distributions are not obtained for arbitrary prior values, but allowing only abundance values contained in the range shown in each window. But within this hypothesis the distributions for the abundances have been obtained without assuming any prior correlation between the individual elements. This is in contrast to previous work (Delahaye \& Pinsonneault 2006; Villante et al. 2014), where abundances of all elements within a group were forced to have the same proportional change. Correlations among the posterior distributions of the abundances appear exclusively as output of the data analysis. For the sake of illustration we provide in Fig. 5 a graphic representation of the correlation among the posterior probability distributions of the different elemental abundances. As expected, the correlations are smaller for the run with the more flexible GP description of the opacity profile uncertainty. But in general for both GP and linear opacity uncertainties, the correlation among the posterior distributions of the abundances included either the $\mathrm{CNO}$ or the meteorite groups are not very large. The exception is provided by the large anticorrelation between the posterior distributions of $\mathrm{C}$ and $\mathrm{O}$ for the analysis with linear opacity uncertainty. We have verified that because the allowed ranges of $\mathrm{C}$ and $\mathrm{O}$ are strongly anticorrelated in this case, the allowed range of CNO group abundance results to be more precise than any of the model priors as seen in the lower central panel in Fig. 4.

The posterior distributions for the other solar input parameters luminosity, diffusion, age, and the eight nuclear rates, are shown in Fig. 6 together with their Gaussian priors. From the figure we see that with the exception of $S_{11}$ and diffusion coefficients, all others parameters do not get significantly modified with respect to the model priors by the inclusion of the neutrino and helioseismic data, irrespective of the form of the opacity uncertainty. We have verified that the helioseismic data - the surface helium abundance $Y_{\mathrm{S}}$ and the location of the bottom of the convective envelope $R_{\mathrm{CZ}}-$ are the most relevant in driving the shift in $S_{11}$. This has to be interpreted as a variation in the effective rate of the proton-proton reaction, but it is not possible to ascribe this change specifically to $S_{11}(0)$ or its derivatives. We see from the figure that the posterior distributions for $S_{11}$ show a preferred value about 1 per cent lower than our prior central value taken from Marcucci, Schiavilla \& Viviani (2013) and 1.5 per cent lower than the newer determination of $S_{11}$ by Acharya et al. (2016). A reassessment of the $\mathrm{p}-\mathrm{p}$ reaction cross-section might be therefore important.

The sound speed data are instead responsible for the preference of lower values of the diffusion coefficients. The reduction in diffusion efficiency that we obtain is in line with previous work (Delahaye \& Pinsonneault 2006; Villante et al. 2014), and it might be related to the occurrence of macroscopic mixing below the convective envelope (Chaboyer et al. 1995; Richard et al. 1996). Our analysis points towards a $30 \pm 10$ percent reduction, larger in comparison with 12 percent found in Villante et al. (2014) and closer to 21 per cent found in Bahcall et al. (2001).

The posterior distributions for the opacity profiles are shown in Fig. 7. In the upper left panel, we plot the $1 \sigma$ range of the intrinsic opacity change $\delta \kappa_{\mathrm{I}}(T)$. This is obtained from the posteriors of the parameters characterizing this function, i.e. the parameters $a$ and $b$ for the linear opacity parametrization given by equation (13), and the 11 values $\delta \kappa_{\mathrm{I} i}=\delta \kappa_{\mathrm{I}}\left(T_{i}\right)$ that sample the function $\delta \kappa_{\mathrm{I}}(r)$ (after marginalizing over the correlation length $L$ ) for GP. By construction, the intrinsic opacity change $\delta \kappa_{\mathrm{I}}(T)$ is defined with respect to a reference opacity calculation $\bar{\kappa}\left(\rho, T, Y, Z_{i}\right)$ that in our analysis include the atomic opacities from OP (Badnell et al. 2005) complemented at low temperatures by molecular opacities from (Ferguson et al. 2005). The fact that the posterior distribution of $\delta \kappa_{\mathrm{I}}(T)$ is not centred at zero (and, moreover, individuates a trend as a function of $T$ ) indicates that there are features of the observational data, namely 

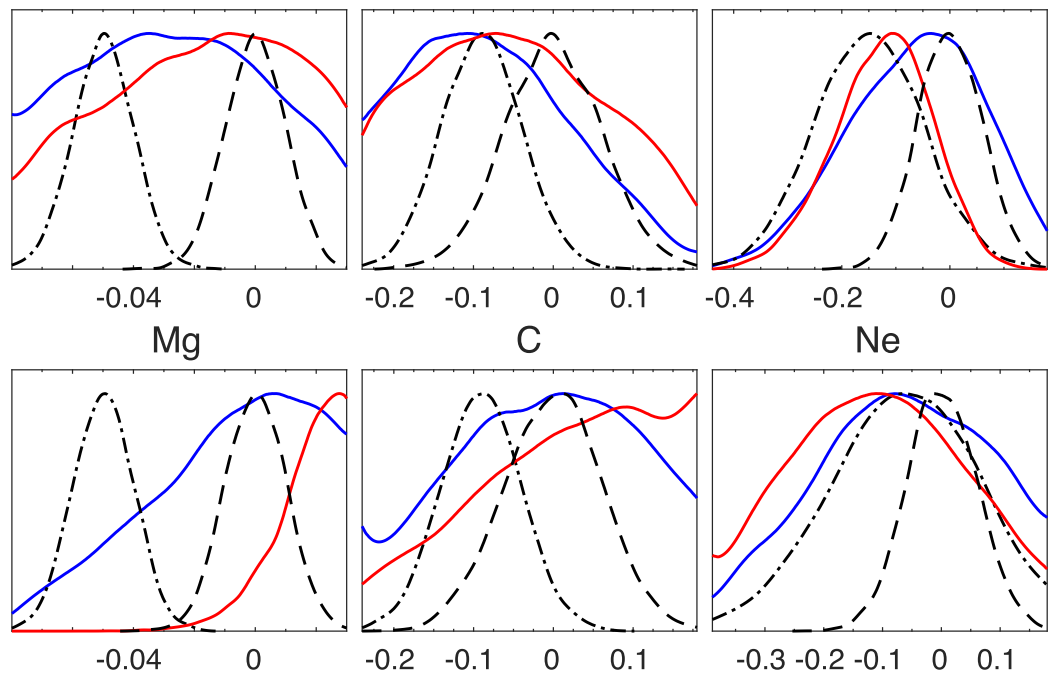

$\mathrm{Si}$

$\mathrm{N}$

$\mathrm{Ar}$
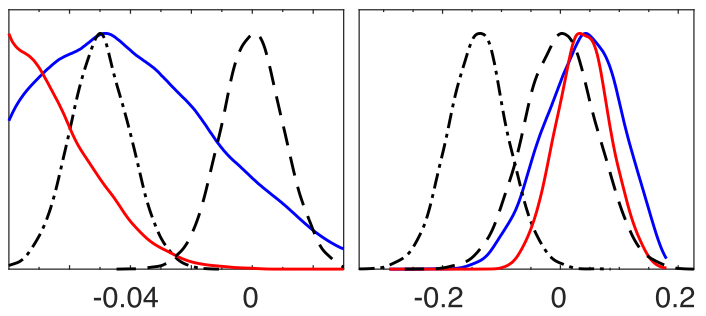

$\mathrm{Fe}$
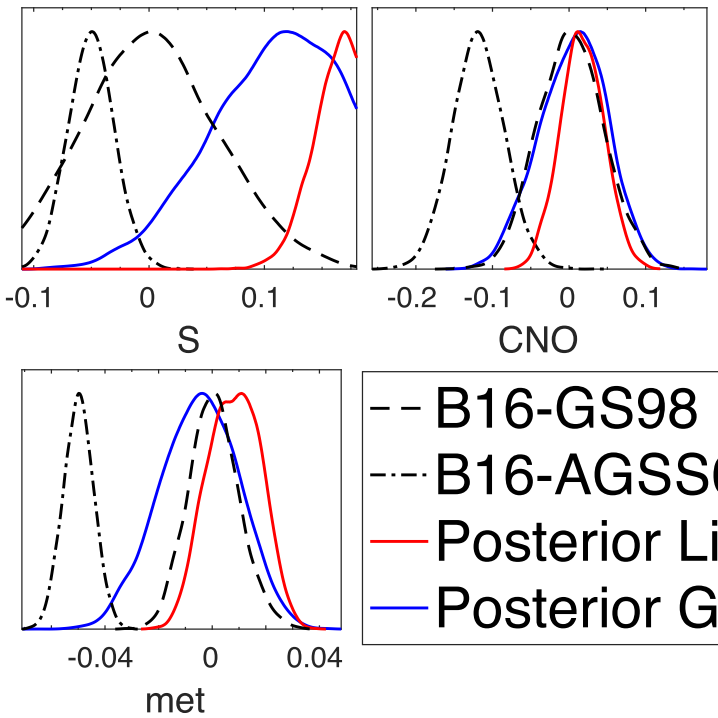

- - B16-GS98 -.-B16-AGSS09-met - Posterior Linear OP Posterior GP OP

Figure 4. Posterior probability distribution for the logarithmic abundances $\left(\varepsilon_{j}-\varepsilon_{j, \mathrm{GS} 98}\right)$ from the analysis of neutrino and helioseismic data with uniform priors for the abundances and for the two choices of the prior opacity uncertainties. The distributions are given in arbitrary units and they have been normalized in such a way that the maximum of all distributions lays at the same height. See the text for details.

the wiggle in the sound speed profile for $0.3<r / \mathrm{R}_{\odot}<0.6$, that cannot be optimally fitted by using the reference opacities, even with the freedom of varying the solar input parameters within their uncertainty ranges and the solar composition in a large intervals considered in this paper, that accommodate both AGSS09met and GS98 observational results. The preference for a slight modification of the OP opacity is consistent with what found in Villante et al. (2014) where indeed it was emphasized that the sound speed is better fitted by using the old OPAL opacity tables.
As explained in Section 3.3, the quantity that is directly constrained by observational data is the SSM opacity profile $\kappa_{\mathrm{SSM}}(T)$, defined according to equation (17), that is affected by composition modifications (and solar model recalibration) in addition to the effects of the intrinsic opacity change $\delta \kappa_{\mathrm{I}}(T)$. In the lower panels of Fig. 7, we show the posterior distributions for $\kappa_{\mathrm{SSM}}(T)$ for the linear (left) and GP (right) description of opacity uncertainty. The posterior distributions for $\kappa_{\mathrm{SSM}}(T)$ are compared with the opacity profiles of B16-GS98 and B16-AGSS09met models. We see that 


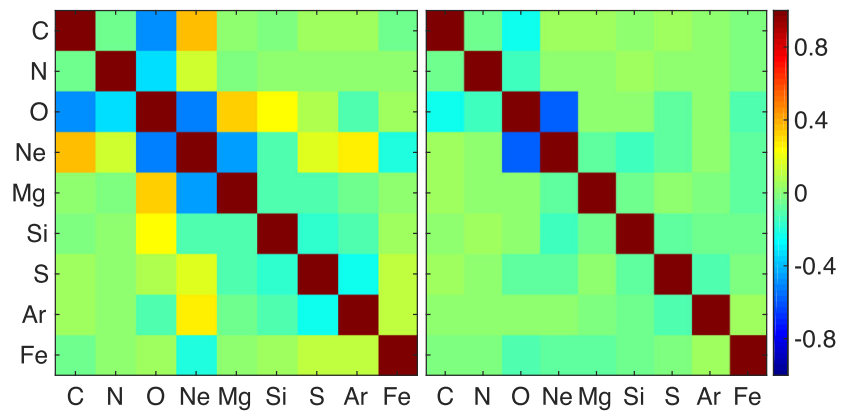

Figure 5. Graphical representation of correlations between the posterior distributions of individual elemental abundances (elements in the same order as in Table 5) for the linear (left) and GP (right) models of intrinsic opacity uncertainty. they are almost coincident with the opacity profile of B16-GS98 model, as it is expected by considering that the best-fitting $\mathrm{CNO}$ and meteoritic elemental abundances, that drive the change in the opacity, are close to GS98 determinations. The optimal opacity profile is well defined by observational data, as it is seen in the central left (right) panels of Fig. 7 where we show the $1 \sigma$ relative dispersion of $\kappa_{\mathrm{SSM}}(r)$ with respect to its mean posterior value. The uncertainty for $\kappa_{\mathrm{SSM}}(r)$ is somewhat larger for the GP opacity uncertainty description, ranging from 0.8 percent at the centre to 4 per cent at the base of the convective envelope, while for the linear uncertainty parametrization it varies from 0.5 per cent to 2.5 per cent.

Finally, we note that the uncertainty in $\kappa_{\mathrm{SSM}}(r)$ is smaller than that of the intrinsic opacity change. In fact, $\delta \kappa_{\mathrm{I}}(r)$ is not directly constrained by the observational properties of the Sun and its determination suffers from the degeneracy with the composition
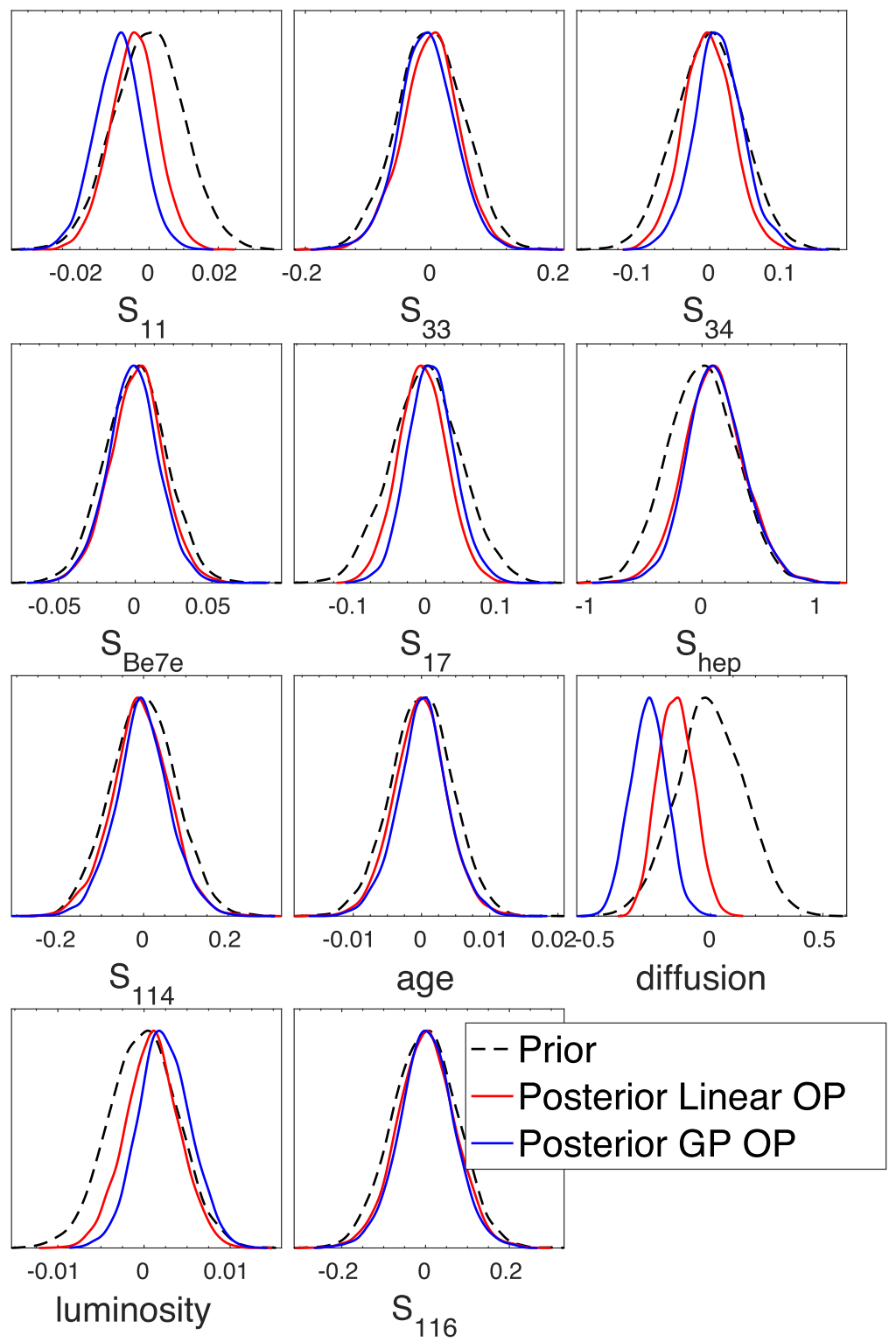

Figure 6. Posterior probability distribution for the Sun luminosity, Sun diffusion, the Sun age, and the eight nuclear rates from the analysis of neutrino and helioseismic data with uniform priors for the abundances and for the two choices of the prior opacity uncertainties. For comparison we also show their prior distribution. The distributions are given in arbitrary units and they have been normalized in such a way that the maximum of all distributions lay at the same height. 

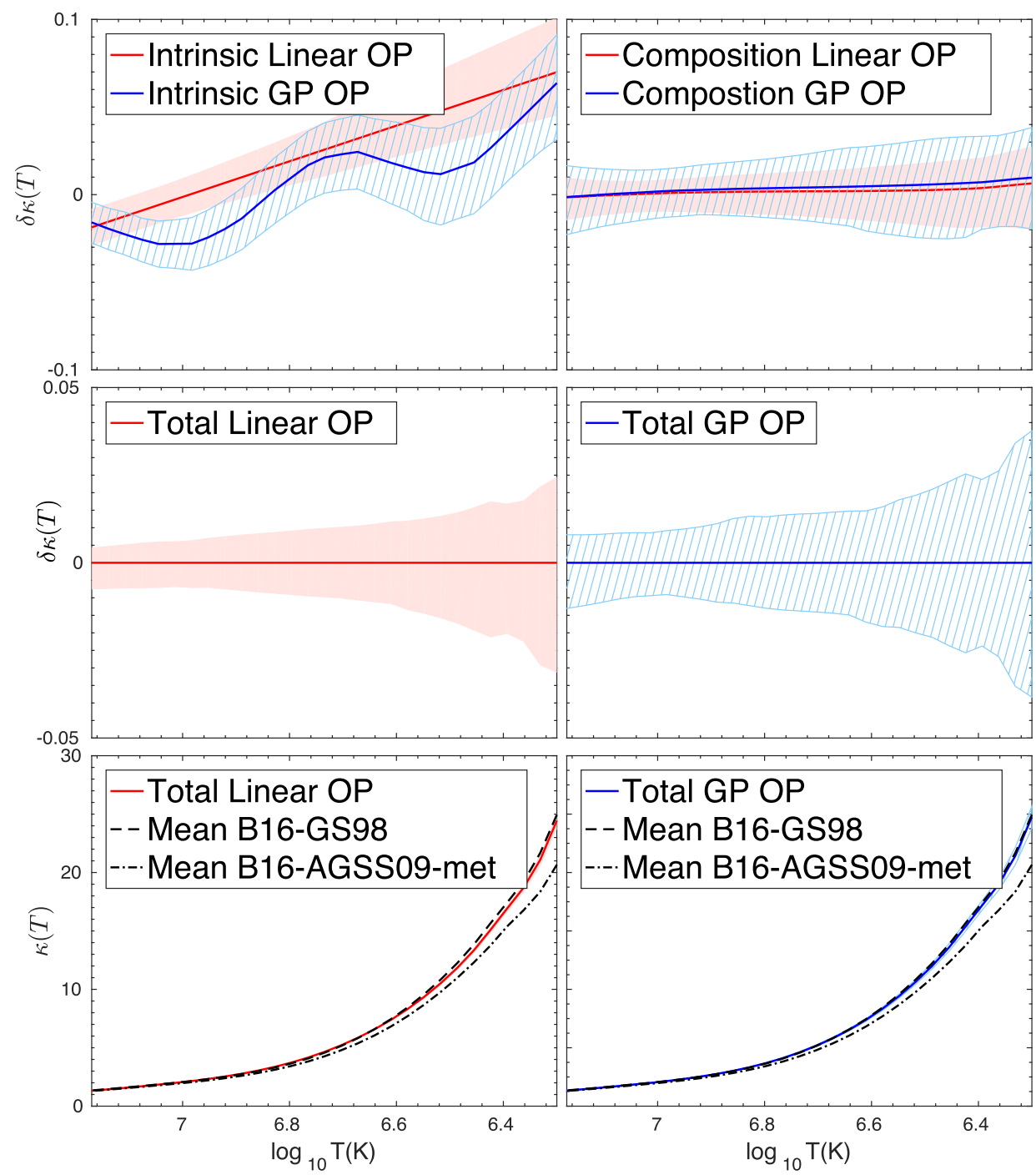

Figure 7. Posterior distribution for the opacity profiles for the analysis with uniform priors for the abundances and the two choices of priors of the opacity uncertainties. See the text for discussion.

opacity change $\delta \kappa_{\mathrm{Z}}(T)$ that is quantified by equation (21). For completeness, we report in the upper (right) panel of Fig. 7, the $1 \sigma$ range for the composition opacity change $\delta \kappa_{\mathrm{Z}}(r)$, obtained from equation (20) with $\delta z_{j}$ being the variance of the posterior distributions of the abundances in Fig. 4 defined relative to the mean of those posteriors. Being defined with respect to the mean of the posterior, the corresponding $\delta \kappa_{\mathrm{Z}}$ are centred around zero.

The result obtained with the uniform composition and with GP opacity uncertainty prior represents our best estimate of the radiative opacity profile in the solar interior. On the other hand, the profiles obtained with other choices of priors, such as the uniform composition with linear opacity uncertainty, or the four cases with B16-GS98 and B16-AGSSmet composition priors with either choice of the opacity uncertainty prior presented in Section 4 , can serve as a measure of the systematic uncertainty in this estimate that reflects dependence on the choice of priors. We show in the top panel in Fig. 8 the $1 \sigma$ range of the posteriors for these six priors. From those we construct a systematic uncertainty in the opacity, at each temperature, defined as the standard deviation of the six reconstructed opacity profiles. The final opacity profile with both error sources added in quadrature is shown in the central panel in Fig. 8 and it ranges from 2 percent at the centre to 7.5 per cent at the bottom of the convective zone.

Finally, for completeness, we show the resulting posterior distribution for the neutrino fluxes in Fig. 9. By construction they constitute the predicted solar neutrino fluxes by models which better describe both the helioseismic and neutrino data. We denote them as helioseismic and neutrino data-driven fluxes, B17-HNDD. We list in Table 6 their best values and $1 \sigma$ uncertainties and in equation (22) their correlations.

$$
\rho=\left(\begin{array}{cccccccc}
1.00 & 0.80 & 0.03 & -0.41 & -0.02 & -0.27 & -0.27 & 0.12 \\
0.80 & 1.00 & 0.06 & -0.33 & -0.05 & -0.28 & -0.29 & 0.01 \\
0.03 & 0.06 & 1.00 & -0.01 & -0.01 & -0.02 & -0.02 & 0.01 \\
-0.41 & -0.33 & -0.01 & 1.00 & 0.13 & -0.03 & -0.02 & -0.03 \\
-0.02 & -0.05 & -0.01 & 0.13 & 1.00 & 0.04 & 0.06 & 0.06 \\
-0.27 & -0.28 & -0.02 & -0.03 & 0.04 & 1.00 & 0.99 & -0.14 \\
-0.27 & -0.29 & -0.02 & -0.02 & 0.06 & 0.99 & 1.00 & -0.12 \\
0.12 & 0.01 & 0.01 & -0.03 & 0.06 & -0.14 & -0.12 & 1.00
\end{array}\right) .
$$



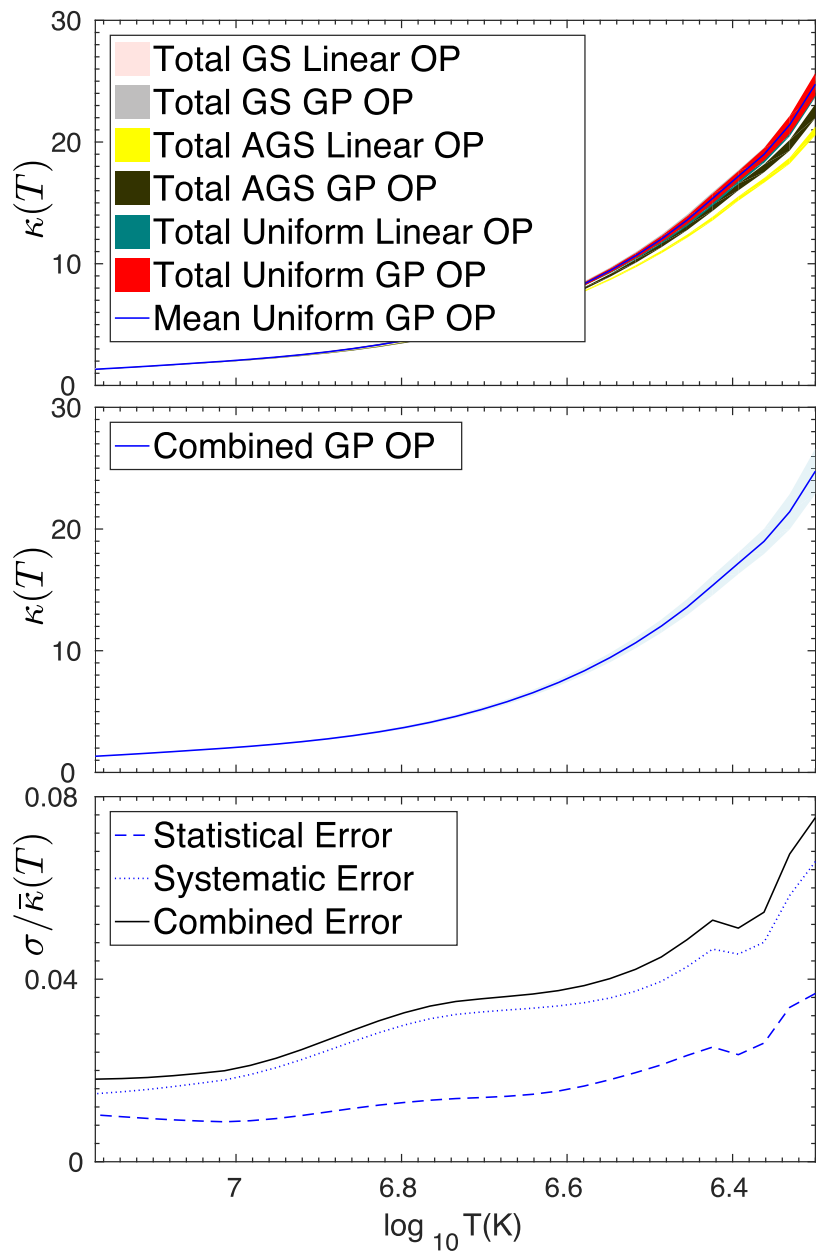

Figure 8. Top: $1 \sigma$ ranges for the posteriors of the opacity profiles corresponding to the six choice of priors for the composition and intrinsic uncertainty variation used in this work. Central: Posterior distribution for the opacity profiles for the analysis with uniform priors for the abundances and the GP opacity uncertainty. The panel shows the mean and $1 \sigma$ range of this distribution combining both statistical and systematic uncertainties. Lower: The panel shows its 'statistical' $1 \sigma$ uncertainty defined as the corresponding variance of the posterior (shown also as 'total' in the central right panel in Fig. 7) and its 'systematic' uncertainty defined as the standard deviation of the six profiles shown on the top window.

As expected, we find that for those neutrino fluxes which are at present most precisely determined in solar neutrino experiments, ${ }^{8} \mathrm{~B}$ and ${ }^{7} \mathrm{Be}$, the $\mathrm{B} 17-\mathrm{HNDD}$ flux is very close to their experimental value used to construct the neutrino data part of the Likelihood function (see last column in Table B1) but with a smaller uncertainty because of the additional indirect constraints imposed by the helioseismic data. Interestingly, we find that with the inclusion of the helioseismic data the precision of the predicted B17-HNDD $\mathrm{CNO}$ fluxes is only at most a factor $\mathcal{O}(2)$ weaker than those of the B16-GS98 or B16-AGSS09met composition models.

\section{SUMMARY}

In this work, we have used Bayesian parameter inference and GP for non-parametric functional reconstruction of the radial opacity profile, with the goal of making an statistically consistent use of the information from helioseismic and neutrino observations for solar modelling. In particular to better determine the solar chemical composition and other solar properties (as well as their uncertainties) which are relevant to the solar composition problem.

In Sections 2 and 3, we have presented a brief summary of the statistical methodology followed and the application of GP for functional reconstruction, and in particular to the radial opacity profile parametrization. Sections 4 and 5 contain our results which we can be summarized as follows:

(i) B16-GS98 versus B16-AGSS09met comparison. This improves over results in Vinyoles et al. (2017) because the linear parametrization of opacities was not flexible enough. Now GP adds more flexibility to the models so our results are now more general and much less dependent on the choice of opacity tables. Helioseismic and neutrino data favours the B16-GS98 model over B16-AGSS09met, but the more flexible modelling of the opacity uncertainty allowed by the GP approach makes this preference less marked.

(ii) Best composition. In our analysis all elements have uncorrelated prior distributions. Therefore, our results are more general than those from previous works (Villante et al. 2014). When considering individual elements, constraints are not very stringent on their abundances. This was expected. The best case is $\mathrm{O}$, with a well-defined Gaussian distribution with $1 \sigma=0.07 \mathrm{dex}$, close to the spectroscopic value. When elements are grouped as CNO or meteoritic, the posterior distributions of these groups are well peaked with uncertainties in the linear(GP) analysis of $0.025(0.045)$ and 0.01(0.015) dex, respectively, comparable to those obtained from spectroscopic measurements. Due to our adoption of a flat prior for elemental abundances and our introduction of the GP approach for modelling opacity uncertainties, our results are quite general, with as little dependence on modelling assumptions as possible (e.g. the bounds in Villante et al. 2014 are obtained in the assumption that the difference OP-OPAL is the measure of the intrinsic opacity uncertainty).

(iii) Non-composition input parameters. The posterior distributions of these parameters have also been determined and are the most general results available to date. $S_{11}$ varies at the $1 \sigma$ level (1 per cent with respect to Marcucci et al. 2013 when compared to Acharya et al. 2016). This is not a large difference, but further work on this important rate might be worth. Our best estimate of the rate of microscopic diffusion is also lower, by about $2 \sigma$, than the standard rate used in solar models. This is qualitatively expected, but the 30 per cent reduction is quantitatively larger than previous estimates that suggested reductions in the range of 15-20 percent (Delahaye \& Pinsonneault 2006; Villante et al. 2014)

(iv) Opacity reconstruction. This is the most important result of our work. We have been able to reconstruct the solar opacity profile in a data-driven way, i.e. without strong assumptions on the solar composition or the underlying opacity tables. Considering uncertainties due to the solar data alone, the opacity uncertainty is about 4 percent at the base of the convective zone and less than 1 per cent at the solar core. Different sets of priors help us quantify a systematic uncertainty in this estimate. From a broad range of assumptions, our more conservative estimate of the total opacity uncertainty (data + priors) is 7.5 per cent at the base of the convective envelope and 1.8 per cent at the solar core.

(v) Neutrino fluxes. We have obtained the posterior distributions of solar $v$-fluxes based on the uniform prior distribution of solar abundances and GP treatment of opacity uncertainties. These fluxes represent the best data-driven reconstruction of the expected solar models $v$-fluxes. For the well-measured ${ }^{8} \mathrm{~B}$ and ${ }^{7} \mathrm{Be}$ fluxes, the final 

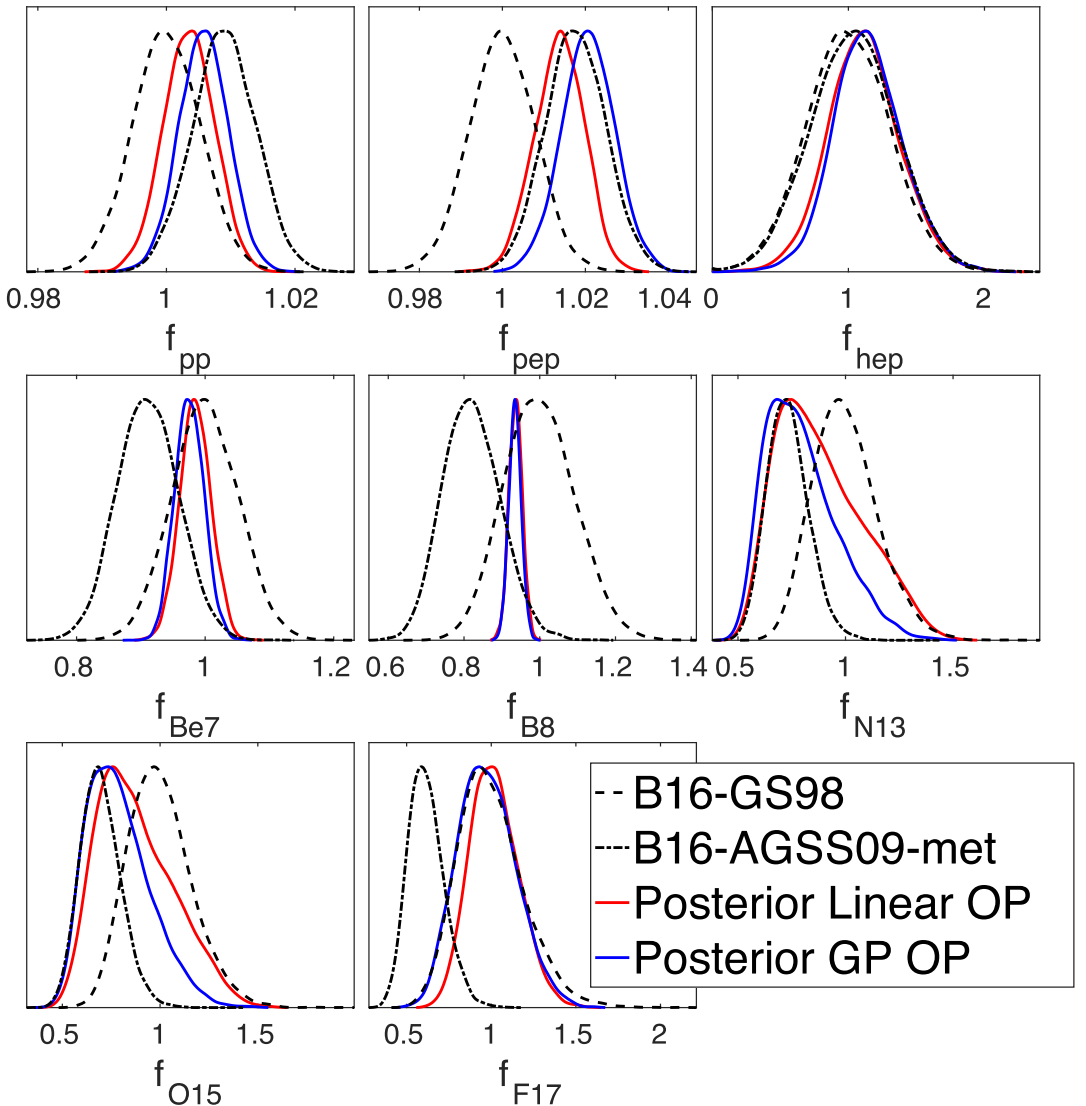

Figure 9. Posterior distributions for the neutrino fluxes for the analysis with uniform priors for the abundances and the two choices of priors of the opacity uncertainties. For the sake of comparison we show the corresponding priors for the B16-GS98 and B16-AGSS09met models. The distributions are given in arbitrary units and they have been normalized in such a way that the maximum of all distributions lay at the same height. The fluxes are shown normalized to the B16-GS98 prediction $f_{i}=\phi_{i} / \phi_{i}^{\mathrm{B} 16-\mathrm{GS} 98}$.

Table 6. Posterior solar neutrino fluxes for uniform-GP models. Units are $10^{10}(\mathrm{pp}), 10^{9}$ $\left({ }^{7} \mathrm{Be}\right), 10^{8}$ (pep, $\left.{ }^{13} \mathrm{~N},{ }^{15} \mathrm{O}\right), 10^{6}\left({ }^{8} \mathrm{~B},{ }^{17} \mathrm{~F}\right)$, and $10^{3}$ (hep) $\mathrm{cm}^{-2} \mathrm{~s}^{-1}$.

\begin{tabular}{lc}
\hline & B17-HNDD $v$-fluxes \\
\hline$\Phi(\mathrm{pp})$ & $6.017\left(1_{-0.0041}^{+0.0033}\right)$ \\
$\Phi($ pep $)$ & $1.470(1 \pm 0.0061)$ \\
$\Phi($ hep $)$ & $9.04\left(1_{-0.21}^{+0.22}\right)$ \\
$\Phi\left({ }^{7} \mathrm{Be}\right)$ & $4.79\left(1_{-0.019}^{+0.027}\right)$ \\
$\Phi\left({ }^{8} \mathrm{~B}\right)$ & $5.10(1 \pm 0.018)$ \\
$\Phi\left({ }^{13} \mathrm{~N}\right)$ & $1.89\left(1_{-0.14}^{+0.32}\right)$ \\
$\Phi\left({ }^{15} \mathrm{O}\right)$ & $1.50\left(1_{-0.20}^{+0.23}\right)$ \\
$\Phi\left({ }^{17} \mathrm{~F}\right)$ & $4.90\left(1_{-0.18}^{+0.22}\right)$ \\
\hline
\end{tabular}

uncertainties reflect experimental uncertainties. For $\mathrm{CN}$ fluxes, the predicted values are approximately only a factor of 2 larger than in the B16 SSMs ( $\sim 20-25$ per cent). This is remarkable because their uncertainty is dominated in our analysis by the $\mathrm{C}+\mathrm{N}$ abundance that has a much larger prior range of variation.

\section{ACKNOWLEDGEMENTS}

We thank C. Peña-Garay and the SOM group (University of Valencia) for their important collaboration in the early stages of this work in particularly for providing us with the computational power to compute the $\mathrm{x} 10000 \mathrm{SSMs}$ calculations. We also thank Michele Maltoni for discussions. We are specially indebted to Johannes Bergstrom for introducing us to the use of Gaussian Processes and MULTINEST and personally initiating this project. This work is supported by USA-NSF grant PHY-1620628, by EU Networks FP10 ITN ELUSIVES (H2020-MSCA-ITN-2015-674896) and INVISIBLES-PLUS (H2020-MSCA-RISE-2015-690575), by MINECO grants FPA2016-76005-C2-1-P and ESP2015-66134-R and ESP2017-82674-R, and by Maria de Maetzu program grant MDM-2014-0367 of ICCUB.

\section{REFERENCES}

Abdurashitov J. N. et al., 2009, Phys. Rev. C, 80, 015807

Abe K. et al., 2011, Phys. Rev. D, 83, 052010

Acharya B., Carlsson B. D., Ekström A., Forssén C., Platter L., 2016, Phys. Lett. B, 760, 584

Adelberger E. G. et al., 2011, Rev. Mod. Phys., 83, 195

Aharmim B. et al., 2013, Phys. Rev. C, 88, 025501

Asplund M., Grevesse N., Sauval J., 2006, Nucl. Phys., A777, 1

Asplund M., Grevesse N., Sauval A. J., Scott P., 2009, Ann. Rev. Astron. Astrophys., 47, 481 (AGSS09)

Badnell N. R., Bautista M. A., Butler K., Delahaye F., Mendoza C., Palmeri P., Zeippen C. J., Seaton M. J., 2005, MNRAS, 360, 458

Bahcall J. N., 1964, Phys. Rev. Lett., 12, 300

Bahcall J. N., Davis R., 1976, Science, 191, 264 
Bahcall J. N., Pinsonneault M. H., 1992, Rev. Mod. Phys., 64, 885 Bahcall J. N., Pinsonneault M. H., 1995, Rev. Mod. Phys., 67, 781 Bahcall J. N., Ulrich R. K., 1988, Rev. Mod. Phys., 60, 297

Bahcall J. N., Bahcall N. A., Shaviv G., 1968, Phys. Rev. Lett., 20, 1209

Bahcall J. N., Pinsonneault M. H., Basu S., 2001, ApJ, 555, 990

Bahcall J. N., Basu S., Pinsonneault M., Serenelli A. M., 2005a, ApJ, 618, 1049

Bahcall J. N., Serenelli A. M., Basu S., 2005b, ApJ, 621, L85

Bahcall J. N., Serenelli A. M., Basu S., 2006, ApJS, 165, 400

Bailey J. E. et al., 2015, Nature, 517, 56

Basu S., Antia H. M., 2008, Phys. Rep., 457, 217

Basu S., Chaplin W. J., Elsworth Y., New R., Serenelli A. M., 2009, ApJ, 699, 1403

Bellini G. et al., 2010, Phys. Rev. D, 82, 033006

Bellini G. et al., 2011, Phys. Rev. Lett., 107, 141302

Bellini G. et al., 2014a, Phys. Rev. D, 89, 112007

Bellini G. et al., 2014b, Nature, 512, 383

Bergström J., Gonzalez-Garcia M. C., Maltoni M., Peña-Garay C., Serenelli A. M., Song N., 2016, J. High Energy Phys., 3, 132

Bottino A., Fiorentini G., Fornengo N., Ricci B., Scopel S., Villante F. L., 2002, Phys. Rev. D, 66, 053005

Caffau E., Ludwig H.-G., Steffen M., Freytag B., Bonifacio P., 2011, Sol. Phys., 268, 255

Castro M., Vauclair S., Richard O., 2007, A\&A, 463, 755

Chaboyer B., Demarque P., Guenther D. B., Pinsonneault M. H., 1995, ApJ, 446, 435

Christensen-Dalsgaard J. et al., 1996, Science, 272, 1286

Cleveland B. T. et al., 1998, ApJ, 496, 505

Colgan J. et al., 2016, ApJ, 817, 116

Cravens J. et al., 2008, Phys. Rev. D, 78, 032002

Delahaye F., Pinsonneault M. H., 2006, ApJ, 649, 529

Deubner F.-L., 1975, A\&A, 44, 371

Ferguson J. W., Alexander D. R., Allard F., Barman T., Bodnarik J. G., Hauschildt P. H., Heffner-Wong A., Tamanai A., 2005, ApJ, 623, 585

Feroz F., Hobson M., 2008, MNRAS, 384, 449

Feroz F., Hobson M., Bridges M., 2009, MNRAS, 398, 1601

Feroz F., Hobson M. P., Cameron E., Pettitt A. N., 2013, preprint (arXiv:1306.2144)

Fiorentini G., Ricci B., Villante F. L., 2001, Phys. Lett. B, 503, 121

Gondolo P., Raffelt G. G., 2009, Phys. Rev. D, 79, 107301

Grevesse N., Sauval A. J., 1998, Space Sci. Rev., 85, 161 (GS98)

Gribov V. N., Pontecorvo B., 1969, Phys. Lett. B, 28, 493

Guzik J. A., Mussack K., 2010, ApJ, 713, 1108

Haxton W. C., Serenelli A. M., 2008, ApJ, 687, 678

Holsclaw T., Alam U., Sanso B., Lee H., Heitmann K., Habib S., Higdon D., 2010a, Phys. Rev. D, 82, 103502

Holsclaw T., Alam U., Sanso B., Lee H., Heitmann K., Habib S., Higdon D., 2010b, Phys. Rev. Lett., 105, 241302

Hosaka J. et al., 2006, Phys. Rev. D, 73, 112001

Iglesias C. A., Rogers F. J., 1996, ApJ, 464, 943

Kaether F., Hampel W., Heusser G., Kiko J., Kirsten T., 2010, Phys. Lett. B, 685, 47

Koshio Y., 2015, AIP Conf. Ser. Vol. 1666, XXVI International Conference on Neutrino Physics and Astrophysics. Am. Inst. Phys., New York, p. 090001

Krief M., Feigel A., Gazit D., 2016, ApJ, 824, 98

Leighton R. B., Noyes R. W., Simon G. W., 1962, ApJ, 135, 474

Mackay D. J. C., 2003, Information Theory, Inference and Learning Algorithms. Cambridge Univ. Press, Cambridge

Marcucci L. E., Schiavilla R., Viviani M., 2013, Phys. Rev. Lett., 110, 192503

Mikheev S. P., Smirnov A. Yu., 1985, Sov. J. Nucl. Phys., 42, 913

Mondet G., Blancard C., Cossé P., Faussurier G., 2015, ApJS, 220, 2

Murphy K. P., 2012, Machine Learning: A Probabilistic Perspective. MIT Press, Cambridge, USA

Noyes R. W., Leighton R. B., 1963, ApJ, 138, 631

Peña-Garay C., Serenelli A., 2008, preprint (arXiv:0811.2424)

Pontecorvo B., 1968, Sov. Phys. JETP, 26, 984
Rasmussen C. E., Williams C. K. I., 2006, Gaussian Processes for Machine Learning. MIT Press, Cambridge, USA

Ricci B., Villante F. L., 2002, Phys. Lett. B, 549, 20

Richard O., Vauclair S., Charbonnel C., Dziembowski W. A., 1996, A\&A, 312,1000

Scott P. et al., 2015a, A\&A, 573, A25

Scott P., Asplund M., Grevesse N., Bergemann M., Sauval A. J., 2015b, A\&A, 573, A26

Seikel M., Clarkson C., Smith M., 2012, J. Cosmol. Astropart. Phys., 1206, 036

Serenelli A., Basu S., Ferguson J. W., Asplund M., 2009, ApJ, 705, L123

Serenelli A. M., Haxton W. C., Pena-Garay C., 2011, ApJ, 743, 24

Serenelli A., Peña-Garay C., Haxton W. C., 2013, Phys. Rev. D, 87, 043001

Tripathy S. C., Christensen-Dalsgaard J., 1998, A\&A, 337, 579

Turck-Chieze S., Cahen S., Casse M., Doom C., 1988, ApJ, 335, 415

Ulrich R. K., 1970, ApJ, 162, 993

Villante F. L., 2010, ApJ, 724, 98

Villante F. L., Serenelli A. M., Delahaye F., Pinsonneault M. H., 2014, ApJ, 787, 13

Vinyoles N., Vogel H., 2016, J. Cosmol. Astropart. Phys., 3, 002

Vinyoles N., Serenelli A., Villante F. L., Basu S., Redondo J., Isern J., 2015, J. Cosmol. Astropart. Phys., 10, 015

Vinyoles N. et al., 2017, ApJ, 835, 202

Wolfenstein L., 1978, Phys. Rev. D, 17, 2369

\section{APPENDIX A: MATCHING METEORITIC AND PHOTOSPHERIC ABUNDANCE SCALES}

Abundances of refractory elements can be determined from the solar spectrum and also from analysis of primitive meteorites. Historically, the latter have proved to be more robust and have been the preferred choice in solar modelling. But element abundances are determined relative to different elements in each case and a matching needs to be applied to bring measurements to the same scale. The process is a simple rigid translation of the meteoritic scale, but the magnitude of this translation can be defined in different ways. Traditionally, two methods have been employed: (1) a shift of the meteoritic scale to match the photospheric silicon abundance and (2) a shift of the meteoritic scale such that the combined (quadratic) difference between photospheric and meteoritic abundances of well-measured refractories is minimized. The uncertainty in this procedure has the potential to impact the true uncertainties in refractory abundances used in solar models. However, we show now here this is a very small uncertainty.

Our approach is to consider the nine more abundant refractory elements that are relevant for radiative opacity calculations, i.e. Na, $\mathrm{Mg}, \mathrm{Al}, \mathrm{Si}, \mathrm{S}, \mathrm{Ca}, \mathrm{Cr}, \mathrm{Fe}$, and $\mathrm{Ni}$. We use the latest photospheric abundances and uncertainties for these elements as given in the most recent revision (Scott et al. 2015a,b) and define the function:

$\chi^{2}(x)=\sum_{j=1,9} \frac{\left(\varepsilon_{j}^{\mathrm{ph}}-\left(\varepsilon_{j}^{\mathrm{met}}+x\right)\right)^{2}}{\sigma_{j}^{2}}$,

where $\sigma_{j}$ is the quadratic combination of the photospheric and meteoritic uncertainties for element $j$ and $x$ is the shift applied to the meteoritic scale and it is defined such that silicon abundances in both scales are equal for $x=0$. Fig. A1 shows $\Delta \chi^{2}(x)=\chi^{2}(x)-$ $\chi_{\min }^{2}(x)$ against $x$ in black solid line for our results using the nine refractories given above and also results when only silicon is used. For the latter, $x_{\min }=0$ by definition. $\chi_{\min }^{2}$ corresponds to $x=0.001$ dex, i.e. the shift in the meteoritic scale is almost the same in this case as in the case of simply matching silicon abundances. Now, considering $\Delta \chi^{2}=1$ we determine the uncertainty in $x$ to 


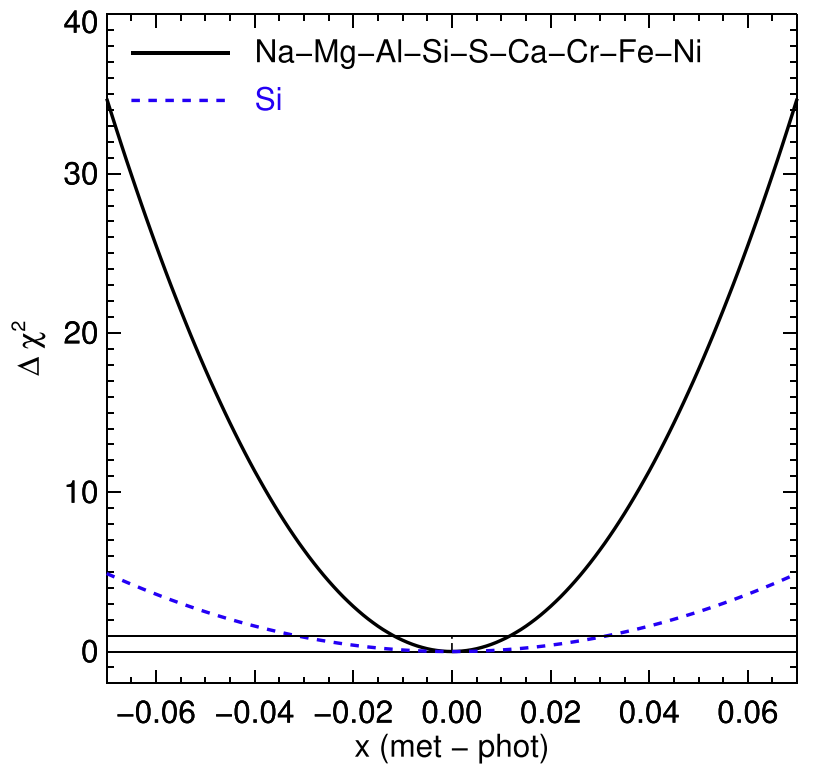

Figure A1. Matching meteoritic and photospheric abundance scales. Black solid line shows results using the nine most abundant refractories. Blue dashed line shows results when using only silicon. Horizontal lines indicate $\Delta \chi^{2}=1$ corresponding to a $1 \sigma$ variation of $x$.

be $1 \sigma=0.012$ dex. This is to be compared with the case when only silicon is used, in which case the uncertainty in $x$ is much larger, $\approx 0.03 \mathrm{dex}$, corresponding to the spectroscopic uncertainty in silicon determination.

From this analysis, we conclude that magnitude of the shift necessary to match the meteoritic and photospheric scales is robustly determined, regardless of whether only silicon or a set of well-measured refractories is used. Also, very importantly, we conclude that the precision with which it is determined is to $0.012 \mathrm{dex}$, much better than that of individual spectroscopic abundance measurements might naively suggest. This uncertainty is comparable to individual meteoritic uncertainties of $\mathrm{Mg}, \mathrm{Si}$, and $\mathrm{Fe}$. While it is possible to include in results presented in Section 4, this would complicate the analysis by introducing a correlation among some element abundances while not having any significant impact in the final results. We have tested that such a correlated uncertainty in the abundances of refractory elements would introduce changes in the predicted values of seismic and neutrino observables much smaller than experimental and other model uncertainties. For the ${ }^{8} \mathrm{~B}$ flux, the most sensitive to refractory abundances, this implies a 2 per cent uncertainty that is negligible compared to the total 12 per cent model uncertainty. As a result, we choose to neglect $x$ as a source of uncertainty in our calculations.

\section{APPENDIX B: DATA INCLUDED IN THE ANALYSIS}

We construct the likelihood function with data from helioseismology and neutrino oscillation experiments. In particular, we include the two helioseismic quantities widely used in assessing the quality of SSMs: the surface helium abundance $Y_{\mathrm{S}}$ and the location of the bottom of the convective envelope $R_{\mathrm{CZ}}$. In Table $\mathrm{B} 1$, we include the experimentally determined value for those two quantities. For illustration we also show the mean and variation of their expected values in the B16 SSMs (which however are not directly use in building the corresponding likelihood). In building the correspond-
Table B1. Main characteristics for the different SSMs with the correspondent model errors and the values for the observational values and their error. For the fluxes units are $10^{10}(\mathrm{pp}), 10^{9}\left({ }^{7} \mathrm{Be}\right), 10^{8}$ (pep, $\left.{ }^{13} \mathrm{~N},{ }^{15} \mathrm{O}\right), 10^{6}\left({ }^{8} \mathrm{~B}\right.$, ${ }^{17} \mathrm{~F}$ ), and $10^{3}$ (hep) $\mathrm{cm}^{2} \mathrm{~s}^{1}$. For the fluxes the last column 'Solar' corresponds to the values obtained from direct fit to the solar neutrino data in Bergström et al. (2016).

\begin{tabular}{lccc}
\hline Qnt. & B16-GS98 & B16-AGSS09met & Solar \\
\hline$Y_{\mathrm{S}}$ & $0.2426 \pm 0.0059$ & $0.2317 \pm 0.0059$ & $0.2485 \pm 0.0035$ \\
$R_{\mathrm{CZ}} / \mathrm{R}_{\odot}$ & $0.7116 \pm 0.0048$ & $0.7223 \pm 0.0053$ & $0.713 \pm 0.001$ \\
$\Phi(\mathrm{pp})$ & $5.98(1 \pm 0.006)$ & $6.03(1 \pm 0.005)$ & $5.971_{(1-0.005)}^{(1+0.006)}$ \\
$\Phi($ pep $)$ & $1.44(1 \pm 0.01)$ & $1.46(1 \pm 0.009)$ & $1.448(1 \pm 0.009)$ \\
$\Phi(\mathrm{hep})$ & $7.98(1 \pm 0.30)$ & $8.25(1 \pm 0.30)$ & $19_{(1-0.47)}^{(1+0.63)}$ \\
$\Phi\left({ }^{7} \mathrm{Be}\right)$ & $4.93(1 \pm 0.06)$ & $4.50(1 \pm 0.06)$ & $4.80_{(1-0.050)}^{(1+0.050)}$ \\
$\Phi\left({ }^{8} \mathrm{~B}\right)$ & $5.46(1 \pm 0.12)$ & $4.50(1 \pm 0.12)$ & $5.16_{(1-0.017)}^{(1+0.025)}$ \\
$\Phi\left({ }^{13} \mathrm{~N}\right)$ & $2.78(1 \pm 0.15)$ & $2.04(1 \pm 0.14)$ & $\leq 13.7$ \\
$\Phi\left({ }^{(5} \mathrm{O}\right)$ & $2.05(1 \pm 0.17)$ & $1.44(1 \pm 0.16)$ & $\leq 2.8$ \\
$\Phi\left({ }^{17} \mathrm{~F}\right)$ & $5.29(1 \pm 0.20)$ & $3.26(1 \pm 0.18)$ & $\leq 85$ \\
\hline
\end{tabular}

ing likelihood function we assume the experimental errors to be totally uncorrelated.

We also include the fractional sound speed differences along the solar radius determined by performing sound speed inversions as described in Basu et al. (2009). The radial resolution of sound speed inversions is typically such that about 70 points are distributed over the solar interior. However, at this resolution the kernels used in the inversions partially overlap for adjacent points. This introduces a correlation between sound speed determinations for which there are no quantifications in the literature. In order to minimize or, at best, avoid this problem we use a coarser resolution with 30 points distributed over $0.06 \leq \mathrm{R}_{\odot} \leq 0.75$. In addition, it is important to note that no information is lost by this reduction in resolution. The reason is that the typical correlation length in solar models is longer than the resolution achieved by using 30 radial points, as described in Section 3.2 for the typical scale over which opacities vary. Therefore, it is the model correlations over the larger scales that dominate the statistical behaviour of the solutions. It is of course also possible to use less than 30 points, i.e. a coarser resolution, but then each radial point would represent a larger fraction of the solar radius, and this would have to be accounted for in the calculation of the $\chi^{2}$ function. This would partly compensate the reduction in the number of degrees of freedom. Moreover, information losses would happen by using e.g. as low as 10 radial points; we would then be underutilizing the available data. In Vinyoles et al (2017) we give a detailed summary of the sources of uncertainties for the sound speed profile. These 'experimental' uncertainties are conservatively assumed to be uncorrelated. For completeness we plot in Fig. B1 the fractional sound speed differences used in our statistical analysis which, by definition, have zero central values.

Finally, we include the results from oscillation experiments in the form of the likelihood of the global analysis of neutrino oscillation data used and described in Bergström et al. (2016) in terms of 3-v oscillations with arbitrary normalization of each of the components of the solar flux. For the sake of illustration we list in the last column in Table B1 the central values and errors of the solar flux normalizations extracted in that analysis. Effectively the effect of the inclusion of the neutrino oscillation data can be understood in terms of a reduced Gaussian likelihood constructed with these extracted 


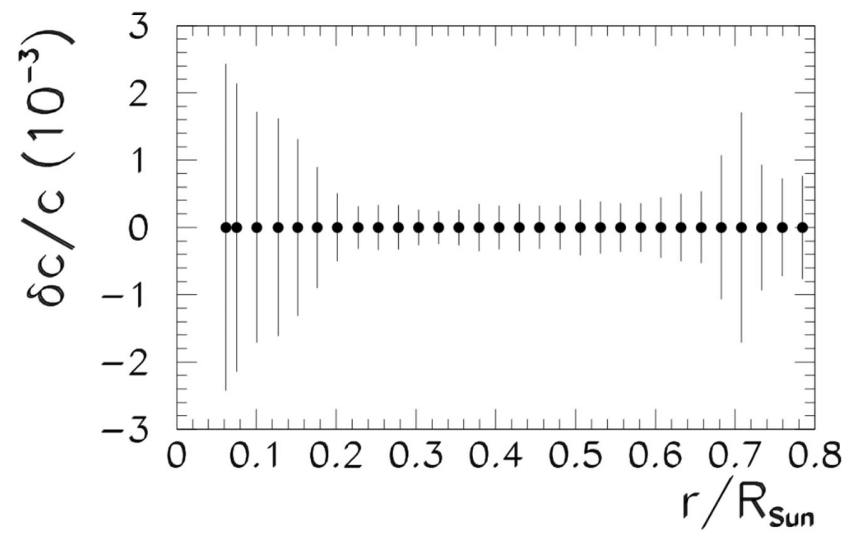

Figure B1. Fractional sound speed data $\delta c / c=\left(c \odot-\bar{c}^{\bmod }\right) / \bar{c}^{\text {mod }}$ used in our analysis.

eight solar fluxes and uncertainties and with the correlation matrix:

$\rho=\left(\begin{array}{cccccccc}1 & 0.99 & -0.05 & 0.08 & -0.14 & -0.20 & -0.19 & -0.11 \\ 0.99 & 1 & -0.05 & 0.08 & -0.14 & -0.20 & -0.19 & -0.11 \\ -0.05 & -0.05 & 1 & -0.08 & 0.10 & -0.01 & -0.00 & -0.00 \\ 0.08 & 0.08 & -0.08 & 1 & -0.17 & -0.31 & 0.09 & 0.10 \\ -0.14 & -0.14 & 0.10 & -0.17 & 1 & -0.02 & -0.03 & -0.01 \\ -0.20 & -0.20 & -0.01 & -0.31 & -0.02 & 0 & 0.18 & 0.09 \\ -0.19 & -0.19 & -0.00 & 0.09 & -0.03 & 0.18 & 1 & 0.36 \\ -0.11 & -0.11 & -0.00 & 0.10 & -0.01 & 0.09 & 0.36 & 1\end{array}\right)$

\section{APPENDIX C: GAUSSIAN PROCESS FOR FUNCTION RECONSTRUCTION}

GP is a non-parametric regression method widely used in statistics and machine learning to reconstruct a function which best describes some data without assuming a parametrization of the function (see e.g. Mackay 2003; Rasmussen \& Williams 2006; Murphy 2012 or the GP webpage ${ }^{2}$ for details). It is used for example in data analysis in cosmology to reconstruct some of the evolution dependent properties (like the dark energy equation of state; Holsclaw et al. 2010a,b; Seikel, Clarkson \& Smith 2012). Seikel et al. (2012) contains a pedagogical description of the process which we briefly sketch here.

The starting assumption is that the value of the function $f$ evaluated at a point $x$ is a Gaussian random variable of mean $\mu(x)$ and variance $\operatorname{Var}(x)$. As the values of the function in two points $x$ and $x^{\prime}$ are not independent, in general one can define a covariant function

\footnotetext{
${ }^{2}$ http://www.gaussianprocess.org/
}

$\operatorname{cov}\left(f(x), f\left(x^{\prime}\right)\right) \equiv C\left(x, x^{\prime}\right)$. The assumed 'prior' covariance function is arbitrary although the obvious hypothesis is that it depends only on the distance between the points. For example, a common choice is a square exponential

$C_{P}\left(x, x^{\prime}\right)=\sigma_{f}^{2} \exp \left(-\frac{\left(x-x^{\prime}\right)^{2}}{2 \lambda^{2}}\right)$,

which depends on the parameters $\sigma_{f}$ and $\lambda$, often referred to as 'hyperparameters' as they do not specify the form of the function but give a measure of its characteristic variations. $\lambda$ can be seen as the characteristic length over which the function changes significantly while $\sigma_{f}$ is its range of variation at each point.

The procedure aims at determining the posterior mean and variance value of the function at some predetermined points, $f_{i}=f\left(x_{i}\right)$, $i=1, N$. This is, to determine $\mu_{i}=\mu\left(x_{i}\right)$ and $C_{i j}=C\left(x_{i}, x_{j}\right)$ starting from some prior mean function $\mu_{p}(x)$ and the chosen prior for the covariant function. It does so by finding the optimum values of $\sigma_{f}$ and $\lambda$ (or marginalizing over them) by confronting them with the data.

In the simplest case, the data to be described corresponds to the value of the function at specific points $\tilde{x}_{a}, y_{a}=f\left(\tilde{x}_{a}\right)$ with $a=1$ to $\tilde{N}$, known with some uncertainties $\sigma_{a}$ (or what is the same with some experimental covariance $\tilde{C}_{a b}$ ). In this case it can be shown that the likelihood for the hyperparameters takes the form

$$
\begin{aligned}
-2 & \ln \mathcal{L}\left(\sigma_{f}, L\right) \\
= & \sum_{a b=1}^{\tilde{N}}\left\{\left(y_{a}-\mu_{a}\right)\left(C_{t}\right)_{a b}^{-1}\left(y_{b}-\mu_{b}\right)+\ln \left(C_{t}\right)_{a b}\right\}+\mathrm{const},
\end{aligned}
$$

where $\mu_{a}=\mu_{p}\left(x_{a}\right)$ and $C_{t}=C_{P}+\tilde{C}$. The posterior mean and covariance for the function at the specific points are

$$
\begin{aligned}
& \bar{f}_{i}=\mu_{p}\left(x_{i}\right)+\sum_{a b=1}^{\tilde{N}} C_{P i a}\left(C_{t}\right)_{a b}^{-1}\left(y_{b}-\mu_{b}\right), \\
& C_{i j}=C_{P i j}-\sum_{a b=1}^{\tilde{N}} C_{P i a}\left(C_{t}\right)_{a b}^{-1} C_{b j} .
\end{aligned}
$$

For the problem at hand, the data - neutrino fluxes, helioseismic data, and sound speeds - are functions of the opacity function that we want to determine (not some values of it) so the procedure to use GP has to be adapted as described in Section 3.2

This paper has been typeset from a $\mathrm{T}_{\mathrm{E}} \mathrm{X} / \mathrm{L} \mathrm{T}_{\mathrm{E}} \mathrm{X}$ file prepared by the author. 